\title{
30. UNDERWAY GEOPHYSICAL MEASUREMENTS: GLOMAR CHALLENGER LEG 74, CAPETOWN TO WALVIS BAY ${ }^{1}$
}

\author{
Philip D. Rabinowitz, ${ }^{2,}{ }^{5}$ Theodore C. Moore, Jr., ${ }^{3}$ Peter E. Borella,${ }^{4}$ and Thomas D. Aitken ${ }^{5}$
}

We present here the navigation and underway geophysical measurements (magnetics, bathymetry, and seismic profiling) obtained aboard Glomar Challenger during Leg 74 of the Deep Sea Drilling Project. On this leg the Glomar Challenger departed Capetown, Republic of South Africa, on 6 June 1980 and proceeded to Site 525 near the crest of Walvis Ridge at about $29^{\circ} \mathrm{S}, 3^{\circ} \mathrm{E}$. After completion of drilling at Site 525 the ship proceeded to Walvis Bay on 22 June 1980 to disembark a sick seaman. The ship departed Walvis Bay the same day and went to Site 526 near the crest of Walvis Ridge. A beacon was dropped at the site, but inclement weather and sea conditions did not permit us to drill at Site $\mathbf{5 2 6}$ at this time. We drilled Sites 527 and 528 on Walvis Ridge with very successful results before returning to drill Site 526, and we completed the cruise with a final hole at Site 529 on Walvis Ridge. The cruise ended in Walvis Bay on 24 July 1980. The ship's track for Leg 74 is shown in Figure 1. Dates and times for the first navigational fix of the day, progressive distance in hundreds of nautical miles along the ship's track, and site numbers are noted. Table 1 lists the positioning information for Leg 74 acquired underway by the satellite navigation system and used to plot the ship's tracks. Errors are generally less than 1 nautical mile (Talwani et al., 1966). Also listed in Table 1 are the regional magnetic field values, computed at each navigation point using the reference field of Fabiano and Peddie (1969), and used to determine the magnetic anomaly profiles in Figure 2.

Figure 2 shows magnetic anomaly and bathymetric data plotted as functions of time, distance, latitude, and longitude, with the distance plotted as a linear function. The data processing procedure, including program listings, is given in Talwani (1969). The vertical scales show

\footnotetext{
${ }^{1}$ Moore, T. C., Jr., Rabinowitz, P. D., et al., Init. Repts. DSDP, 74; Washington (U.S. Govt, Publishing Co.).

2 Department of Oceanography, Texas A\&M University, College Station, Texas 77843.

3 Exxon Production Research Laboratory, Houston, Texas 77001. 92093 .

4 Deep Sea Drilling Project, Scripps Institution of Oceanography, La Jolla, California
}

depth in uncorrected fathoms (assuming a sound speed of $800 \mathrm{fm} / \mathrm{s}$ or $1463 \mathrm{~m} / \mathrm{s}$ ) under "D" and magnetic anomaly values in gammas under "M." On the lowermost scale at the bottom of the figure, distances are shown at intervals of 200 nautical miles. In addition, tick marks shown above the distance scale indicate the distance at which any change in course or speed occurred. The corresponding course and speed between changes and the coordinates at the points of change are noted above the distance scale listings. Navigational changes, which occur too frequently to be shown in the space available, or minor adjustments in course or speed, are indicated only by tick marks. At the top of the figures, from top to bottom, are shown date in day-monthyear, time in hours, with labels every $4 \mathrm{hr}$, and latitude and longitude in degrees, with a tick mark whenever a whole degree is crossed.

Seismic reflection profile records for Leg $\mathbf{7 4}$ are given in Figure 3. These data were recorded on dry-paper EDO recorders and in general were obtained using two air guns (20 and 40 in. $^{3}$ firing chambers) as a sound source. Depths are labeled on the sides of the records in two-way reflection time ( $1 \mathrm{~s} \approx 750 \mathrm{~m}$ water depth). Times and dates are listed on the bottom of the records to key into the navigation plots (Fig. 1) and listings (Table 1).

For detailed descriptions of the geophysical measurements in the vicinity of Sites 325 to 329 the reader is referred to Rabinowitz and Simpson (this volume).

\section{ACKNOWLEDGMENTS}

The efforts of the laboratory technicians aboard Glomar Challenger, Leg 74, under the direction of laboratory officer Ted Gustafson, are greatly appreciated.

\section{REFERENCES}

Fabiano, E. G., and Peddie, N. U., 1969. Grid values of total magnetic intensity I.G.R.F., 1965. U.S. ESSA Tech. Rept., 38:55.

Talwani, M., 1969. A Computer System for the Reduction, Storage, and Display of Underway Data Acquired at Sea. Lamont-Doherty Geological Observatory of Columbia University Tech. Rept. 1, CU-1-69 N00014-67-A-0108-0004.

Talwani, M., Dorman, J., Worzel, J. L., and Bryan, G. M., 1966. Navigation at sea by satellite. J. Geophys. Res., 71:5891-5902. 
Table 1. Positioning information (from satellite navigation system): Leg 74.

\begin{tabular}{|c|c|c|c|c|c|c|c|}
\hline $\begin{array}{c}\text { Date } \\
\text { (day/mo./y.) }\end{array}$ & Time & $\begin{array}{l}\text { Latitude } \\
\text { (South) }\end{array}$ & $\begin{array}{l}\text { Longitude } \\
\text { (East) }\end{array}$ & Miles & Speed & Heading & $\begin{array}{c}\text { Regional } \\
\text { Magnetic } \\
\text { Field Values }\end{array}$ \\
\hline $6-6-80$ & 2035 & 3332.84 & 1736.57 & 0.0 & 7.4 & 310 & 28363. \\
\hline $6-6-80$ & 2045 & 3332.05 & 1735.44 & 1.2 & 8.9 & 311 & 28364. \\
\hline $6-6-80$ & 2055 & 3331.07 & 1734.09 & 2.7 & 8.8 & 302 & 28365 . \\
\hline $6-6-80$ & 2105 & 3330.29 & 1732.60 & 4.2 & 9.0 & 289 & 28366. \\
\hline $6-6-80$ & 2155 & 3327.85 & 1724.08 & 11.7 & 10.6 & 286 & 28365 . \\
\hline $6-6-80$ & 2215 & 3326.85 & 1720.00 & 15.3 & 9.6 & 286 & 28364. \\
\hline $7-6-80$ & 0005 & 3321.95 & 16.59 .79 & 32.8 & 9.5 & 294 & 28360 . \\
\hline $7-6-80$ & 0305 & 3310.23 & 1628.61 & 61.4 & 9.5 & 293 & 28364. \\
\hline $7-6-80$ & 0605 & 3258.75 & $15 \$ 7.44$ & 89.9 & 9.5 & 293 & 28368. \\
\hline $7-6-80$ & 0615 & 3258.13 & 1555.72 & 91.5 & 9.4 & 300 & 28368 . \\
\hline $7-6-80$ & 0625 & 3257.35 & 1554.10 & 93.1 & 9.9 & 291 & 28369. \\
\hline $7-6-80$ & 0655 & 3255.54 & 1548.62 & 98.0 & 9.5 & 332 & 28369. \\
\hline $7-6-80$ & 0705 & 3254.13 & 1547.76 & 99.6 & 9.8 & 337 & 28372 . \\
\hline $7-6-80$ & 0715 & 3252.63 & 1547.00 & 101.2 & 9.7 & 341 & 28375 . \\
\hline $7-6-80$ & 0725 & 3251.09 & 1546.39 & 102.8 & 9.6 & 347 & 28379. \\
\hline $7-6-80$ & 0855 & 3237.06 & 1542.65 & 117.2 & 7.0 & 306 & 28412. \\
\hline $7-6-80$ & 0905 & 3236.37 & 1541.54 & 118.4 & 9.2 & 264 & 28413. \\
\hline $7-6-80$ & 1015 & 3237.50 & 1528.85 & 129.1 & 7.5 & 304 & 28400 . \\
\hline $7-6-80$ & 1025 & 3236.80 & 1527.63 & 130.4 & 9.8 & 340 & 28401. \\
\hline $7-6-80$ & 1045 & 3233.72 & 1526.33 & 133.7 & 7.5 & 304 & 28408 . \\
\hline $7-6-80$ & 1055 & 3233.01 & 1525.12 & 134.9 & 9.5 & 264 & 28409 . \\
\hline $7-6-80$ & 1355 & 3235.97 & 1451.32 & 163.5 & 9.9 & 269 & 28375 . \\
\hline $7-6-80$ & 1405 & 3235.99 & 1449.36 & 165.2 & 10.3 & 290 & 28373. \\
\hline $7-6-80$ & 1705 & 3224.95 & 1415.04 & 196.2 & 10.0 & 290 & 28376. \\
\hline $7-6-80$ & 2005 & 3214.70 & 1341.81 & 226.1 & 9.8 & 291 & 28377. \\
\hline $7-6-80$ & 2035 & 3212.88 & 1336.40 & 231.0 & 9.3 & 318 & 28377. \\
\hline $7-6-80$ & 2045 & 3211.72 & 1335.18 & 232.5 & 9.4 & 323 & 28380 . \\
\hline $7-6-80$ & 2105 & $32 \quad 9.20$ & 1332.97 & 235.7 & 9.7 & 291 & 28385 . \\
\hline $8-6-80$ & 0005 & 3158.47 & $13 \quad 1.03$ & 264.8 & 9.4 & 294 & 28388. \\
\hline $8-6-80$ & 0305 & 3146.59 & 1230.80 & 293.1 & 9.5 & 295 & 28396. \\
\hline $8-6-80$ & 0415 & 3141.86 & 1219.06 & 304.1 & 9.5 & 298 & 28399. \\
\hline $8-6-80$ & 0615 & 3132.82 & 1159.51 & 323.1 & 9.4 & 293 & 28408 . \\
\hline $8-6-8 C$ & 0755 & 3126.60 & 1142.63 & 338.7 & 9.4 & 289 & 28411. \\
\hline $8-6-80$ & 1055 & 3117.35 & 1111.55 & 366.8 & 9.1 & 287 & 28410 . \\
\hline $8-6-80$ & 1115 & 3116.44 & 118.15 & 369.9 & 8.8 & 285 & 28410 . \\
\hline $8-6-80$ & 1415 & $31 \quad 9.41$ & 1038.29 & 396.4 & 9.2 & 287 & 28403. \\
\hline $8-6-80$ & 1445 & 318.04 & 1033.18 & 401.0 & 9.4 & 293 & 28402 . \\
\hline $8-6-80$ & 1535 & $31 \quad 4.97$ & 1024.73 & 408.8 & 9.2 & 307 & 28403. \\
\hline $8-6-80$ & 1545 & 314.05 & 1023.30 & 410.4 & 9.3 & 312 & 28405 . \\
\hline $8-6-80$ & 1615 & $\begin{array}{ll}31 & 0.88\end{array}$ & 1019.30 & 415.0 & 9.6 & 291 & 28410 . \\
\hline $8-6-80$ & 1915 & 3050.60 & 948.05 & 443.7 & 10.0 & 294 & 28410 . \\
\hline $8-6-80$ & 2155 & 3029.81 & 919.83 & 470.3 & 9.4 & 299 & 28414 . \\
\hline $9-6-80$ & 0025 & 3028.31 & 855.98 & 493.8 & 9.6 & 293 & 28424 . \\
\hline $9-6-80$ & 0235 & 3020.02 & 833.88 & 514.6 & 9.5 & 290 & 28425 . \\
\hline $9-6-80$ & 0405 & 3015.05 & 818.47 & 528.8 & 8.2 & 291 & 28424. \\
\hline $9-6-80$ & 0505 & 3012.04 & 89.70 & 537.0 & 10.8 & 287 & 28423 . \\
\hline $9-6-80$ & 0615 & $30 \quad 8.35$ & 755.70 & 549.6 & 9.2 & 285 & 28419. \\
\hline $9-6-80$ & 0915 & $\begin{array}{ll}30 & 1.00\end{array}$ & 724.97 & 577.2 & 9.3 & 284 & 28407. \\
\hline $9-6-80$ & 1205 & 2954.53 & 655.47 & 603.6 & 9.3 & 289 & 28393. \\
\hline $9-6-80$ & 1215 & 2954.02 & 653.79 & 605.1 & 9.2 & 297 & 28393. \\
\hline $9-6-80$ & 1225 & 2953.32 & 652.21 & 606.6 & 9.3 & 302 & 28393. \\
\hline $9-6-80$ & 1455 & 2940.96 & 629.51 & 629.9 & 9.2 & 292 & 28403. \\
\hline $9-6-80$ & 1505 & 2940.37 & 627.88 & 631.4 & 9.6 & 283 & 28403. \\
\hline $9-6-80$ & 1805 & 2933.87 & 555.46 & 660.4 & 9.9 & 283 & 28384. \\
\hline $9-6-80$ & 2105 & 2927.04 & 522.28 & 690.0 & 10.2 & 282 & 28363. \\
\hline $10-6-80$ & 0005 & 2920.45 & 448.16 & 720.5 & 10.2 & 282 & 28340 . \\
\hline $10-6-80$ & 0135 & 2917.17 & 431.07 & 735.7 & 10.1 & 276 & 28327. \\
\hline $10-6-80$ & 0415 & 2914.05 & $\begin{array}{ll}4 & 0.27\end{array}$ & 762.8 & 10.0 & 275 & 28297. \\
\hline $10-6-80$ & 0445 & 2913.61 & 354.56 & 767.8 & 10.3 & 280 & 28290. \\
\hline $10-6-80$ & 0745 & 298.06 & 319.69 & 798.7 & 10.4 & 279 & 28260 . \\
\hline $10-6-80$ & 0855 & $\begin{array}{lll}29 & 6.02\end{array}$ & 36.07 & 810.8 & 8.1 & 285 & 28247. \\
\hline $10-6-80$ & 0935 & 294.55 & 30.11 & 816.2 & 5.7 & 291 & 28243. \\
\hline $10-6-80$ & 0945 & $29 \quad 4.20$ & 259.09 & 817.2 & 0.0 & 360 & 28243. \\
\hline $10-6-80$ & 0955 & $29 \quad 4.20$ & 259.09 & 817.2 & 0.0 & 360 & 28243. \\
\hline Site 525 & & & & & & & \\
\hline $19-6-80$ & 0615 & $29 \quad 4.20$ & 259.09 & 817.2 & 7.7 & 94 & 28241. \\
\hline $19-6-80$ & 0625 & $\begin{array}{ll}29 & 4.29\end{array}$ & 30.55 & 818.4 & 6.7 & 84 & 28243. \\
\hline $19-6-80$ & 0705 & $\begin{array}{ll}29 & 3.87\end{array}$ & 35.65 & 822.9 & 7.8 & 71 & 28250 . \\
\hline $19-6-80$ & 0715 & $\begin{array}{ll}29 & 3.47\end{array}$ & 37.06 & 824.2 & 9.3 & 66 & 28253. \\
\hline $19-6-80$ & 1015 & 2852.36 & 336.32 & 852.1 & 9.3 & 66 & 28323. \\
\hline $19-6-80$ & 1305 & 2842.06 & $\begin{array}{ll}4 & 3.82\end{array}$ & 878.3 & 9.8 & 74 & 28389. \\
\hline $19-6-80$ & 1425 & 2838.64 & 418.12 & 891.3 & 9.7 & 76 & 28417. \\
\hline $19-6-80$ & 1605 & 2834.94 & 435.97 & 907.4 & 9.5 & 68 & 28452 . \\
\hline $19-6-80$ & 1705 & 2831.49 & 446.06 & 917.0 & 9.0 & 65 & 28475 . \\
\hline $19-6-80$ & 1745 & 2829.05 & 452.27 & 922.9 & 9.3 & 70 & 28490 . \\
\hline $19-6-80$ & 2045 & 2819.53 & 522.26 & 951.0 & 9.4 & 70 & 28558. \\
\hline $19-6-80$ & 2345 & $\begin{array}{ll}28 & 9.92\end{array}$ & 552.52 & 979.3 & 9.5 & 71 & 28626. \\
\hline $20-6-80$ & 0245 & $\begin{array}{ll}28 & 0.75\end{array}$ & 623.15 & 1007.8 & 9.2 & 72 & 28693 . \\
\hline $20-6-80$ & 0545 & 2752.41 & 652.84 & 1035.4 & 9.2 & 69 & 28757 . \\
\hline $20-6-80$ & 0845 & 2742.63 & 721.94 & 1062.9 & 9.1 & 67 & 28825 . \\
\hline $20-6-80$ & 1145 & 2732.34 & 750.35 & 1090.1 & 8.9 & 66 & 28894. \\
\hline $20-6-80$ & 1235 & 2729.36 & 758.03 & 1097.5 & 9.0 & 57 & 28913. \\
\hline $20-6-80$ & 1245 & 2728.55 & 759.47 & 1099.0 & 9.3 & so & 28917. \\
\hline $20-6-80$ & 1545 & 2710.81 & 823.49 & 1126.8 & 9.4 & 52 & 29006. \\
\hline $20-6-80$ & 1845 & 2653.71 & 848.48 & 1154.9 & 9.4 & 52 & 29094. \\
\hline $20-6-80$ & 2105 & 2640.42 & $\begin{array}{ll}9 & 7.96\end{array}$ & 1176.8 & 9.9 & 55 & 29163. \\
\hline $20-6-80$ & 0005 & 2623.57 & 935.44 & 1206.6 & 9.9 & 54 & 29255. \\
\hline $21-6-80$ & 0125 & 2615.87 & 947.30 & 1219.7 & 9.9 & 49 & 29296. \\
\hline $21-6-80$ & 0425 & 2556.65 & 1012.32 & 1249.3 & 9.9 & 49 & 29394. \\
\hline $21-6-80$ & 0725 & 2537.25 & 1037.21 & 1278.9 & 9.9 & 48 & 29493. \\
\hline
\end{tabular}

Table 1. (Continued).

\begin{tabular}{|c|c|c|c|c|c|c|c|}
\hline $\begin{array}{c}\text { Date } \\
\text { (day/mo./y.) }\end{array}$ & Time & $\begin{array}{l}\text { Latitude } \\
\text { (South) }\end{array}$ & $\begin{array}{c}\text { Longitude } \\
\text { (East) }\end{array}$ & Miles & Speed & Heading & $\begin{array}{c}\text { Regional } \\
\text { Magnetic } \\
\text { Field Values }\end{array}$ \\
\hline \multicolumn{8}{|c|}{ Site 525 (Cont.) } \\
\hline $21-6-80$ & 0855 & 2527.37 & 1049.44 & 1293.7 & 9.2 & 43 & 29543. \\
\hline $21-6-80$ & 1105 & 2512.98 & 114.58 & 1313.6 & 9.1 & 52 & 29612. \\
\hline $21-6-80$ & 1355 & 2457.36 & 1127.29 & 1339.4 & 9.2 & 57 & 29696. \\
\hline $21-6-80$ & 1655 & 2442.33 & i1 52.82 & 1367.0 & 9.4 & 56 & 29782. \\
\hline $21-6-80$ & 1955 & 2426.77 & 1218.48 & 1395.1 & 9.3 & 53 & 29869. \\
\hline $21-6-80$ & 2235 & 2412.09 & 1240.56 & 1420.0 & 9.8 & 57 & 29949. \\
\hline $21-6-80$ & 2335 & $24 \quad 6.84$ & 1249.58 & 1429.7 & 9.7 & 50 & 29979. \\
\hline $22-6-80$ & 0035 & $24 \quad 0.70$ & 1257.84 & 1439.4 & 9.7 & 45 & 30011 . \\
\hline $22-6-80$ & 0135 & 2353.93 & $13 \quad 5.42$ & 1449.1 & 9.3 & 39 & 30045. \\
\hline $22-6-80$ & 0325 & 2340.78 & 1317.26 & 1466.2 & 9.0 & 47 & 30107. \\
\hline $22-6-80$. & 0345 & 2338.75 & 1319.69 & 1469.2 & 9.1 & 54 & 30118. \\
\hline $22-6-80$ & 0525 & 2329.94 & 1333.13 & 1484.3 & 4.5 & 55 & 30166. \\
\hline \multicolumn{8}{|l|}{ Walvis Bay } \\
\hline $22-6-80$ & 2315 & 2331.43 & 1334.57 & 1487.1 & 4.8 & 236 & 30162. \\
\hline $23-6-80$ & 2335 & 2332.33 & 1333.11 & 1488.7 & 7.9 & 231 & 30157. \\
\hline $23-6-80$ & 0115 & 2340.60 & 1321.98 & 1501.9 & 8.2 & 257 & 30113. \\
\hline $23-6-80$ & 0125 & 2340.90 & 1320.53 & 1503.2 & 8.8 & 267 & 30110. \\
\hline $23-6-80$ & 0135 & 2340.97 & 1318.92 & 1504.7 & 8.5 & 259 & 30108. \\
\hline $23-6-80$ & 0145 & 2341.24 & 1317 & 1506.1 & 8.1 & 245 & 30105. \\
\hline $23-6-80$ & 0155 & 2341 & 131 & 1507.5 & 7.9 & 231 & 30102. \\
\hline $23-6-80$ & 0235 & 2345.11 & 1311.61 & 1512.7 & 7.5 & 221 & 30084. \\
\hline $23-6-80$ & 0425 & 2355.46 & & 1526.5 & 7.6 & 226 & 30035. \\
\hline $23-6-80$ & 0635 & $24 \quad 6.77$ & 1248.73 & 1542.9 & 6.1 & 227 & \\
\hline $23-6-80$ & 0645 & $24 \quad 7.46$ & 1247.91 & 1543.9 & 6.2 & & \\
\hline $23-6-80$ & 0905 & 2416.39 & 1235.37 & 1558.4 & 7.7 & 229 & \\
\hline $23-6-80$ & 1205 & 2431.28 & 1216.03 & 1581.5 & 8.1 & 231 & 29849. \\
\hline $23-6-80$ & 1505 & 2446.50 & 1155.31 & 1605.7 & 8.2 & 227 & 29769. \\
\hline $23-6-80$ & 1545 & 2450.18 & 1150.83 & 1611.2 & 8.2 & 226 & 29750. \\
\hline $23-6-80$ & 1755 & $25 \quad 2.44$ & 1136.50 & 1629.0 & 8.4 & 230 & 29689. \\
\hline 23-6. & 1935 & 2511 & 112 & 1643.0 & 8.2 & 227 & 29642 . \\
\hline 23-6. & 2025 & 2515 & 18 & & 8.5 & 232 & 29619. \\
\hline $23-6-80$ & 2205 & 2524 . & $\begin{array}{ll}11 & 6.52\end{array}$ & 166 & 7.6 & 222 & 29572. \\
\hline $23-6-80$ & 2235 & 2527.50 & $\begin{array}{ll}11 & 3.69\end{array}$ & $\begin{array}{l}160 \\
166\end{array}$ & 8.2 & 230 & 29559. \\
\hline $24-6-80$ & 0115 & 2541.57 & 1044.97 & 1689.9 & 8.1 & 226 & 294 \\
\hline $24-6-80$ & 0415 & 2558.36 & 1025.60 & 1714.1 & 8.1 & 228 & \\
\hline $24-6-80$ & 0525 & $\begin{array}{lll}26 & 4.64\end{array}$ & 1017.71 & 1723.6 & 8.5 & 234 & 29372. \\
\hline $24-6-80$ & 0545 & $\begin{array}{ll}26 & 6.29\end{array}$ & 1015.16 & 1726.4 & 8.5 & 227 & 29363. \\
\hline $24-6-8$ & 0845 & 2623.62 & 954. & 1751 & 8.7 & 228 & 29277. \\
\hline 24 & 1145 & 2640.80 & 932. & 1777 & 9.0 & 229 & 29191. \\
\hline & 1445 & 2658 . & 99. & 1804.9 & 9.5 & 231 & 29103. \\
\hline $24-6-80$ & 1745 & 2716. & 844. & 1833.5 & 9.5 & 231 & 29013. \\
\hline $24-6-80$ & 2025 & 2731. & 822.06 & $\begin{array}{l}1858 . \\
.\end{array}$ & 8.4 & 231 & 28933. \\
\hline $\begin{array}{l}-6-80 \\
-6-80\end{array}$ & 2105 & 2735.41 & 817.13 & 186 & 9.6 & 232 & 28916. \\
\hline $24-6$. & 2325 & 2749.07 & 757.19 & 1886.7 & 9.7 & 242 & 288 \\
\hline & 01 & 2759 & & & 9.4 & 240 & 287 \\
\hline & 02 & 284 & 725 & 191 & & 235 & \\
\hline & 0455 & 2815 & 76 & 193 & 9.4 & 238 & 287 \\
\hline $25-6$ - & 0755 & 2830. & 635 & & 9. & 23 & \\
\hline $25-6-8$ & 0915 & 2837. & 6 & 197 & 8.8 & 23 & \\
\hline 25 & 1055 & 2845 . & 613. & 1994.3 & 8.3 & 238 & \\
\hline $25-6$. & 1335 & 2857. & 552. & 2016.4 & 8.2 & 243 & 28482. \\
\hline $25-6$ & 1635 & 298. & 526 & 2041.1 & 8.1 & 244 & 28419 . \\
\hline $25-6-80$ & 1745 & 2912 . & 517. & 2050.5 & 7.0 & 243 & 28395 . \\
\hline & 193 & 2918 & 53 & 206 & 6.7 & 237 & 28363. \\
\hline & & 2921 . & 458 & & 7.4 & 246 & 28348. \\
\hline & & & & & & 24 & \\
\hline & & & & & 6. & 250 & \\
\hline & 03 & & & & 6. & & \\
\hline $26-6-$ & 0645 & 2951. & & & 6. & & \\
\hline$-6-80$ & 0945 & $\begin{array}{lll}30 & 0.85\end{array}$ & 324 & 216 & 6. & 242 & 281 \\
\hline 26 & 1035 & $\begin{array}{ll}30 & 3.44\end{array}$ & 318.22 & 216 & 7. & 250 & 281 \\
\hline $26-6$. & 1105 & $30 \quad 4.76$ & 313.97 & 2169.8 & 7.7 & 241 & 28099. \\
\hline $26-6-80$ & 1145 & $\begin{array}{ll}30 & 7.19\end{array}$ & 38.75 & 2174.9 & 2.8 & 241 & 28087. \\
\hline
\end{tabular}

First attempt at Site $\$ 26$

\begin{tabular}{|c|c|c|c|c|c|c|c|c|}
\hline $27-6-80$ & 1935 & 30 & 9.11 & $\begin{array}{l}35.05 \\
3\end{array}$ & 2178.7 & 0.1 & 248 & 28076. \\
\hline $27-6-80$ & 1945 & 30 & 9.12 & 5.03 & 2178.7 & 5.9 & 329 & 28076. \\
\hline $27-6-80$ & 1955 & 30 & 8.27 & $\begin{array}{ll}3 & 4.44\end{array}$ & 2179.7 & 8.9 & 330 & 28078. \\
\hline $27-6-80$ & 2055 & 30 & 0.50 & 259.33 & 2188.6 & 9.3 & 336 & 28091. \\
\hline $27-6-80$ & 2255 & & 43.38 & 250.93 & 2207.2 & 9.5 & 330 & 28125. \\
\hline $27-6-80$ & 2355 & & 35.07 & 245.52 & 2216.8 & 9.6 & 326 & 28139. \\
\hline $28-6-80$ & 0035 & & 29.76 & 241.41 & 2223.2 & 9.5 & 329 & 28148. \\
\hline $28-6-80$ & 0045 & & 28.39 & 240.49 & 224.8 & 9.5 & 333 & 28150. \\
\hline $28-6-80$ & 0225 & 291 & 14.17 & 232.39 & 2240.7 & 9.7 & 331 & 28177. \\
\hline $28-6-80$ & 0525 & & 48.72 & 216.30 & 2269.7 & 9.8 & 329 & 28223. \\
\hline $28-6-80$ & 0825 & & 23.38 & 159.29 & 2299.1 & 10.1 & 326 & 28267. \\
\hline $28-6-80$ & 1045 & 28 & 3.71 & 144.51 & 2322.7 & 6.7 & 347 & 28299. \\
\hline $28-6-80$ & 1105 & 28 & 1.54 & 143.97 & 2325.0 & 4.1 & 117 & 28304. \\
\hline $28-6-80$ & 1115 & 28 & 1.85 & 144.66 & 2325.6 & 5.5 & 123 & 28304. \\
\hline $28-6-80$ & 1125 & 28 & 2.35 & 145.53 & 2326.6 & 1.7 & 122 & 28304. \\
\hline $28-6-80$ & 1135 & 28 & 2.50 & 145.80 & 2326.8 & 0.0 & 217 & 28304. \\
\hline \multicolumn{9}{|l|}{ Site 527} \\
\hline $4 \cdot 7 \cdot 80$ & 0600 & 28 & 2.75 & 1.45 .57 & 2327.2 & 6.5 & 218 & 28302. \\
\hline $4-7-80$ & 0620 & 28 & 4.46 & 144.05 & 2329.3 & 4.5 & 161 & 28295. \\
\hline $4-7-80$ & 0630 & 28 & 5.17 & 144.32 & 2330.1 & 4.9 & 145 & 28293. \\
\hline $4.7-80$ & 0640 & 28 & 5.84 & 144.84 & 2330.9 & 6.7 & 141 & 28292. \\
\hline
\end{tabular}


Table 1. (Continued).

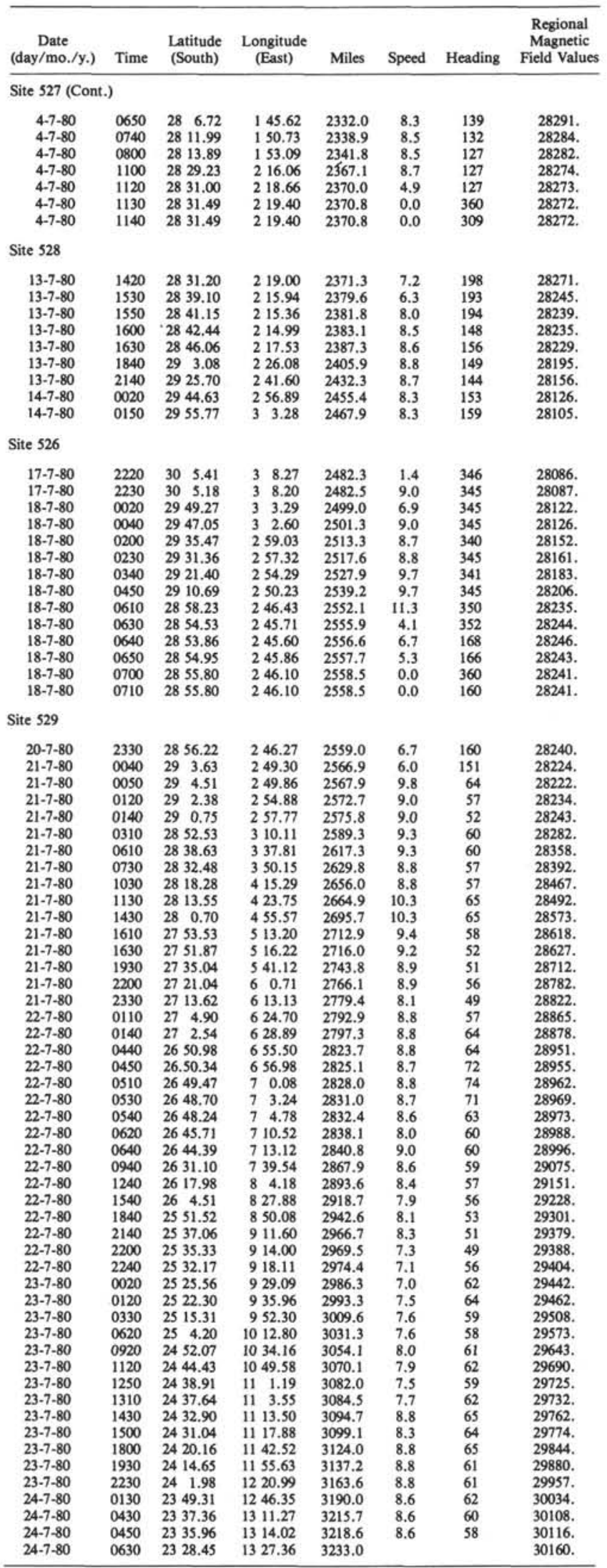


P. D. RABINOWITZ, T. C. MOORE, JR., P. E. BORELLA, T. D. AITKEN

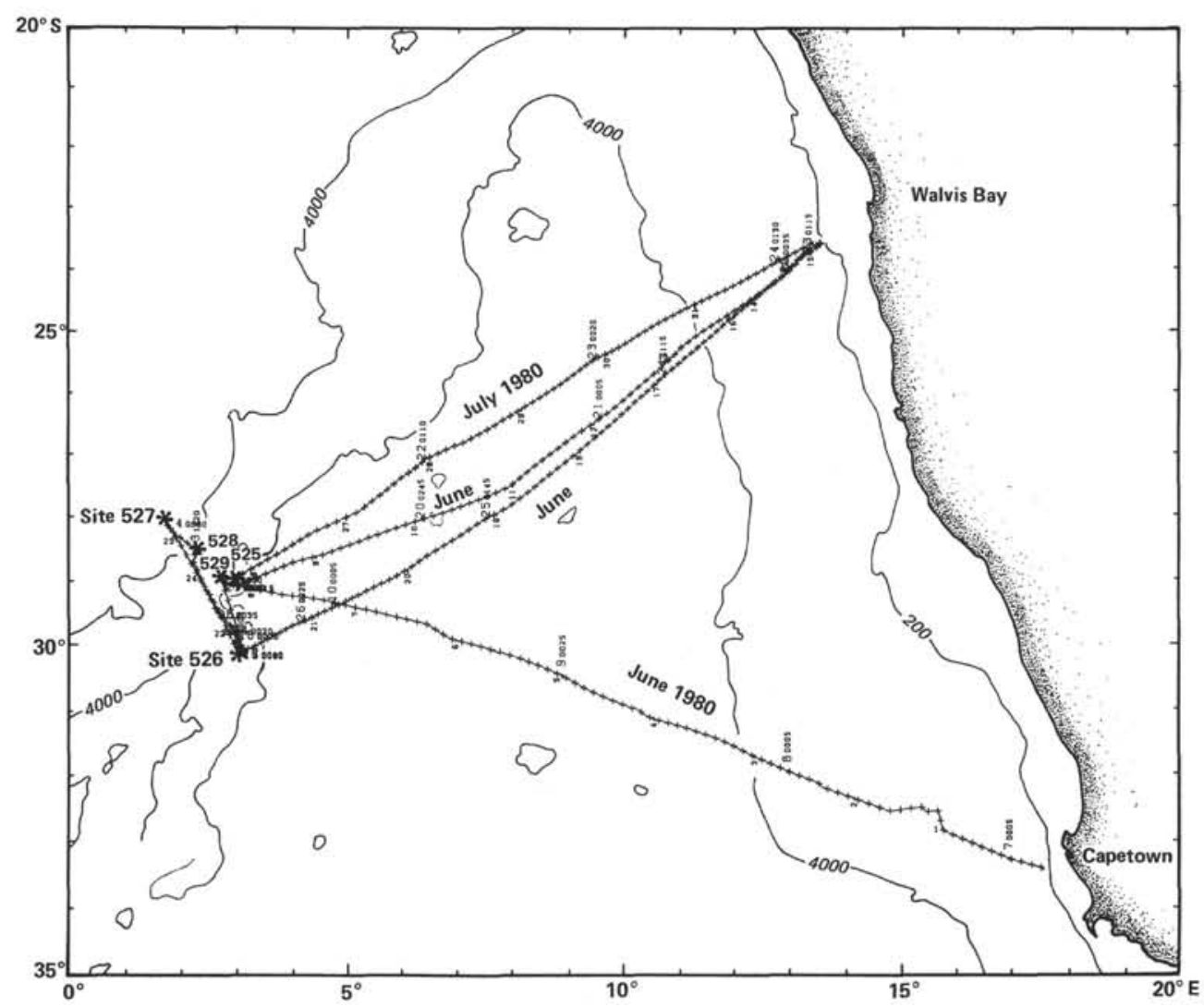

Figure 1. Track chart of Glomar Challenger Leg 74 from Capetown to Walvis Bay. First navigation fix of every day and distance in hundreds of nautical miles along track are shown. Hour marks along track designated by "plus" marks. 


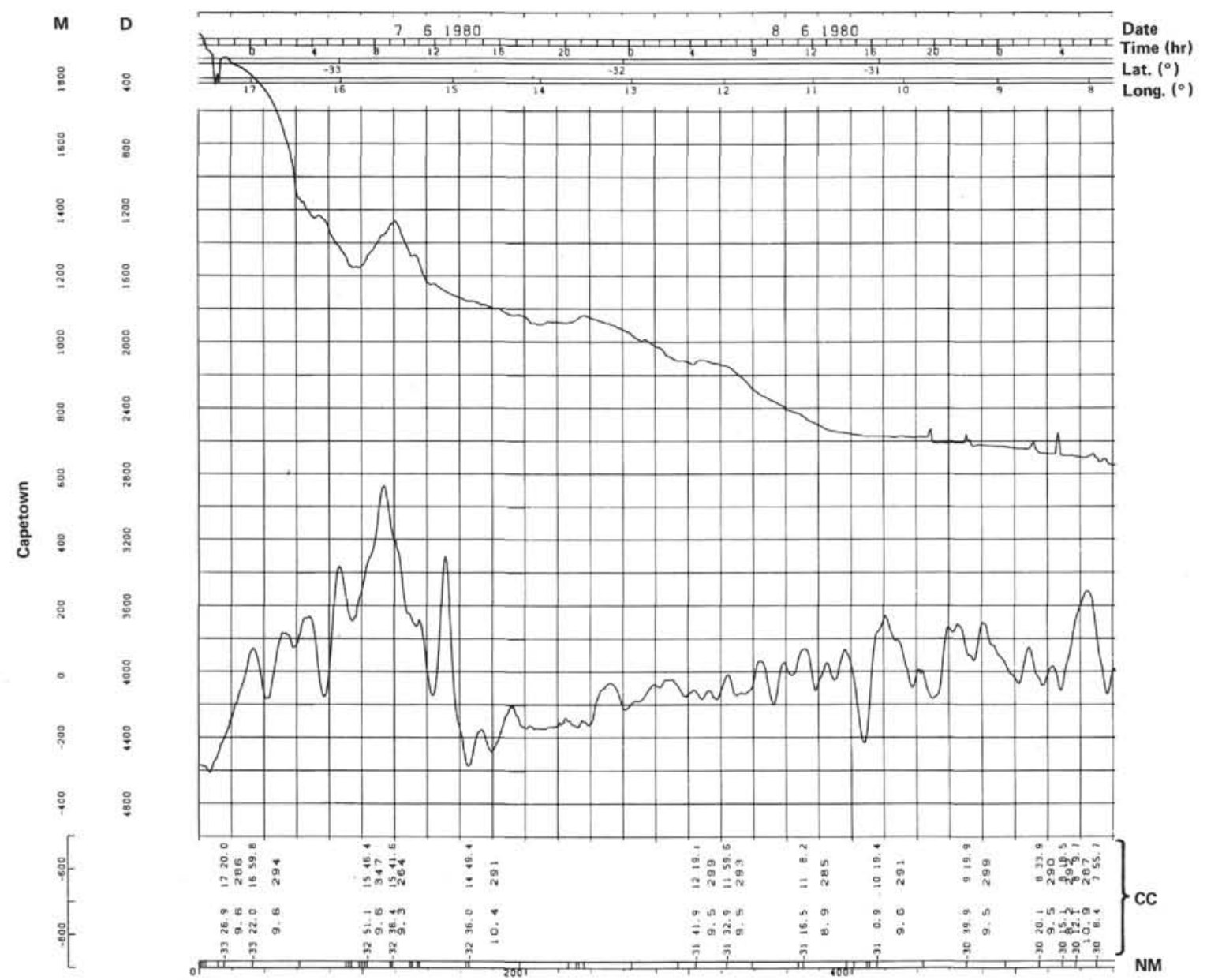

Figure 2. Magnetic anomaly and bathymetric profiles along track of Leg 74 of Glomar Challenger. Plots and scales further explained in text. $(\mathrm{D}=$ depth, in uncorrected fathoms. $\mathrm{M}=$ magnetic anomaly values, in gammas; $\mathrm{NM}=$ distance, in nautical miles; $\mathrm{CC}=$ course changes, speed between changes, and coordinates at the point of change.) 


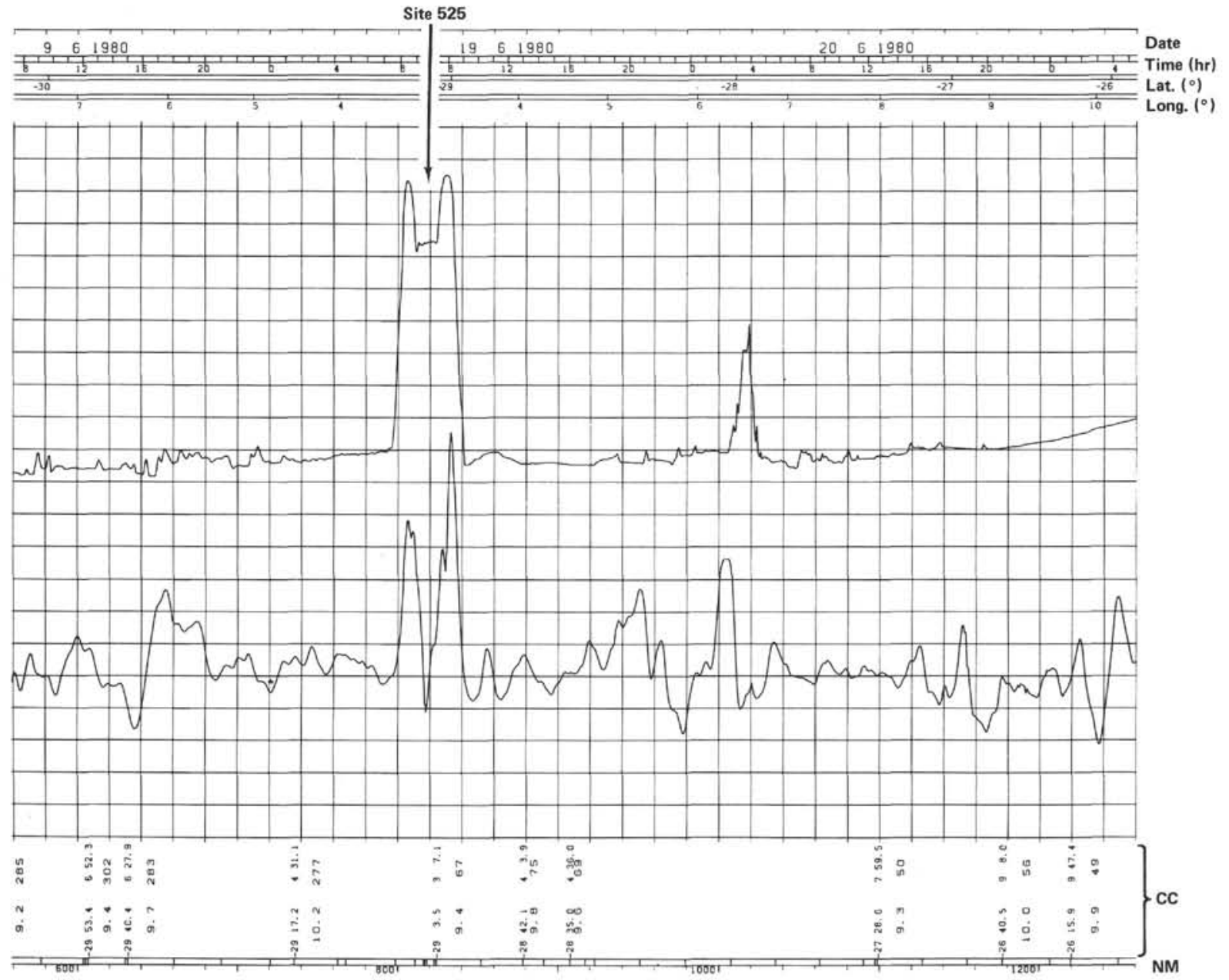

Figure 2. (Continued). 


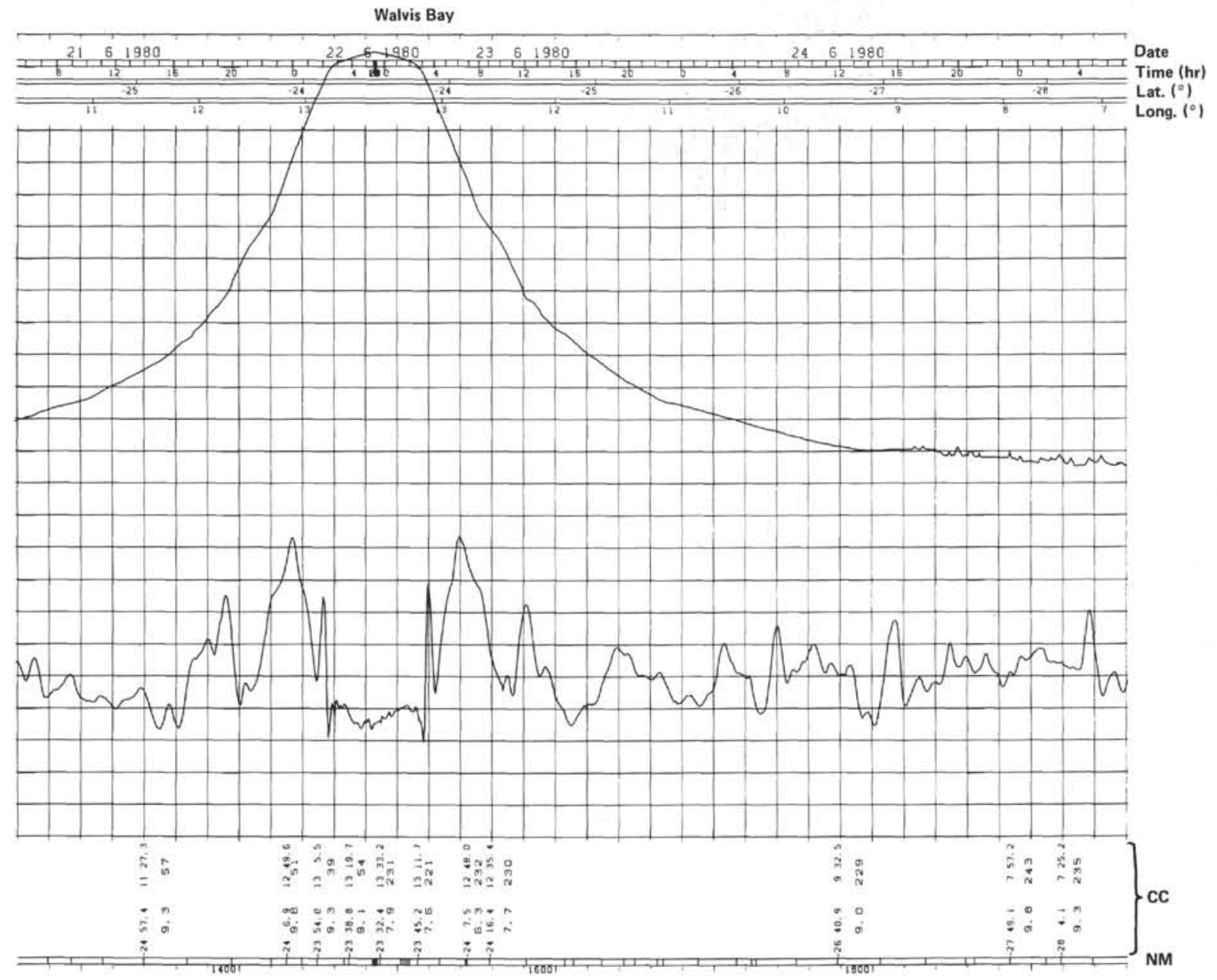

Figure 2. (Continued). 
P. D. RABINOWITZ, T. C. MOORE, JR., P. E. BORELLA, T. D. AITKEN

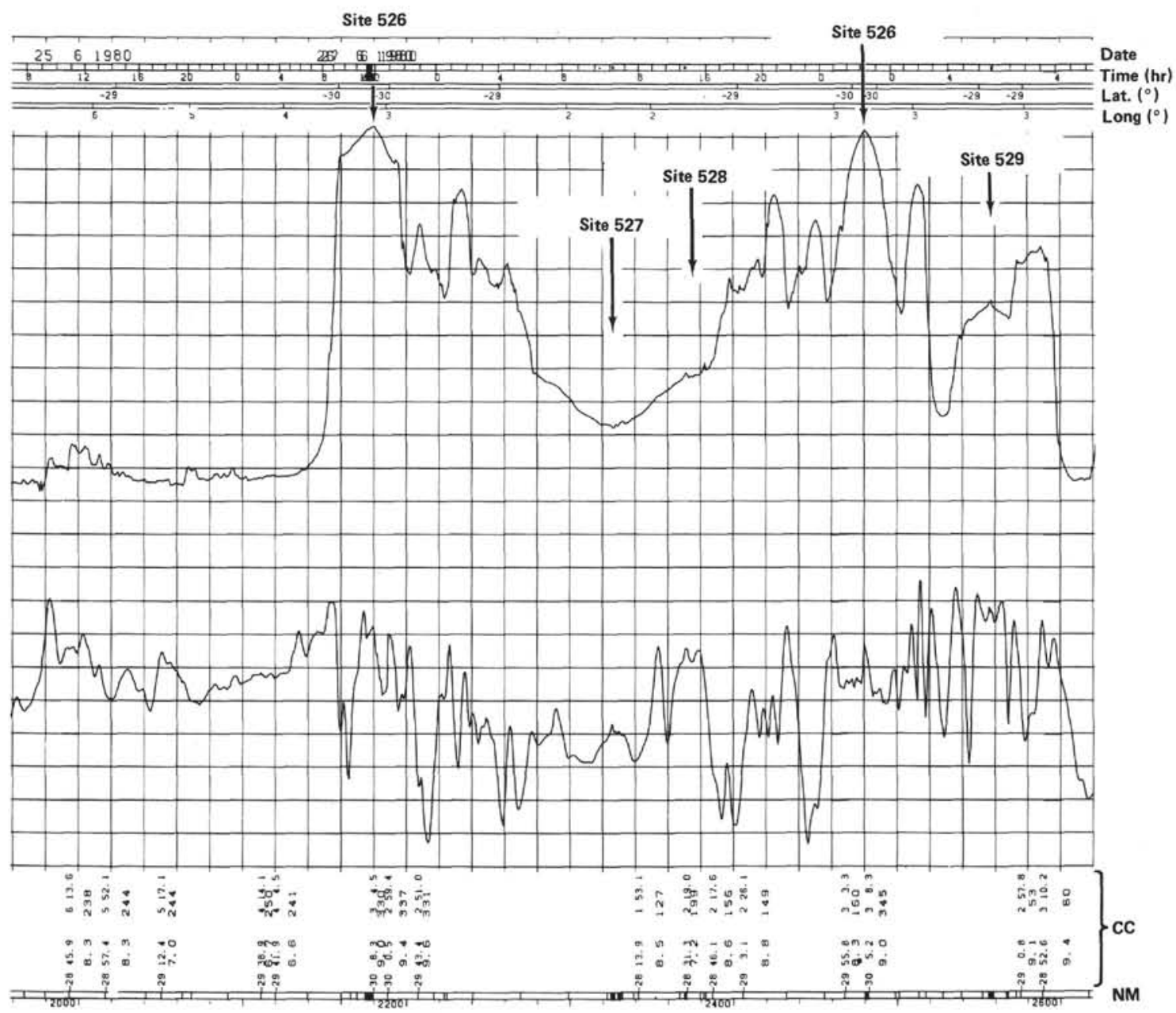

Figure 2. (Continued). 


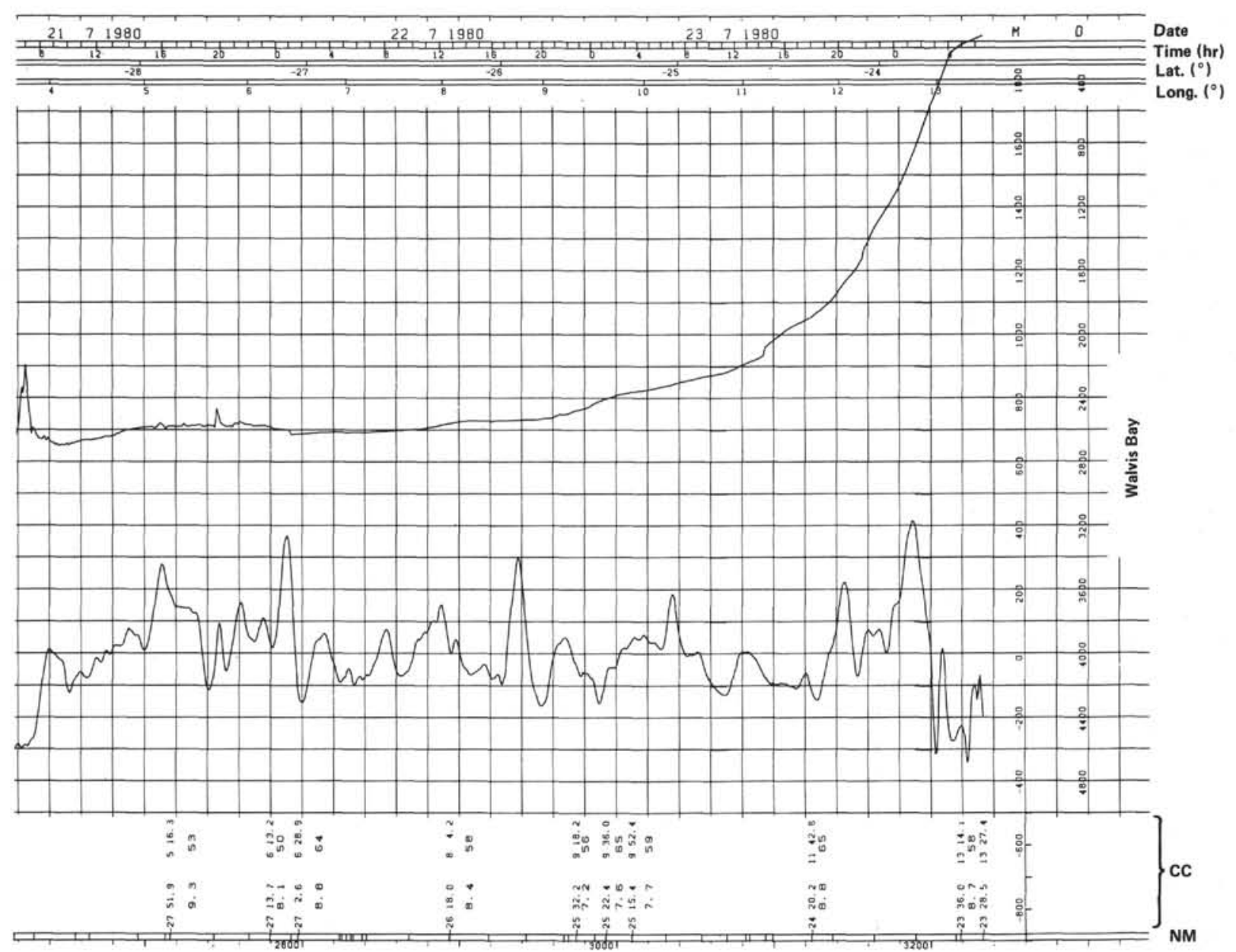

Figure 2. (Continued). 


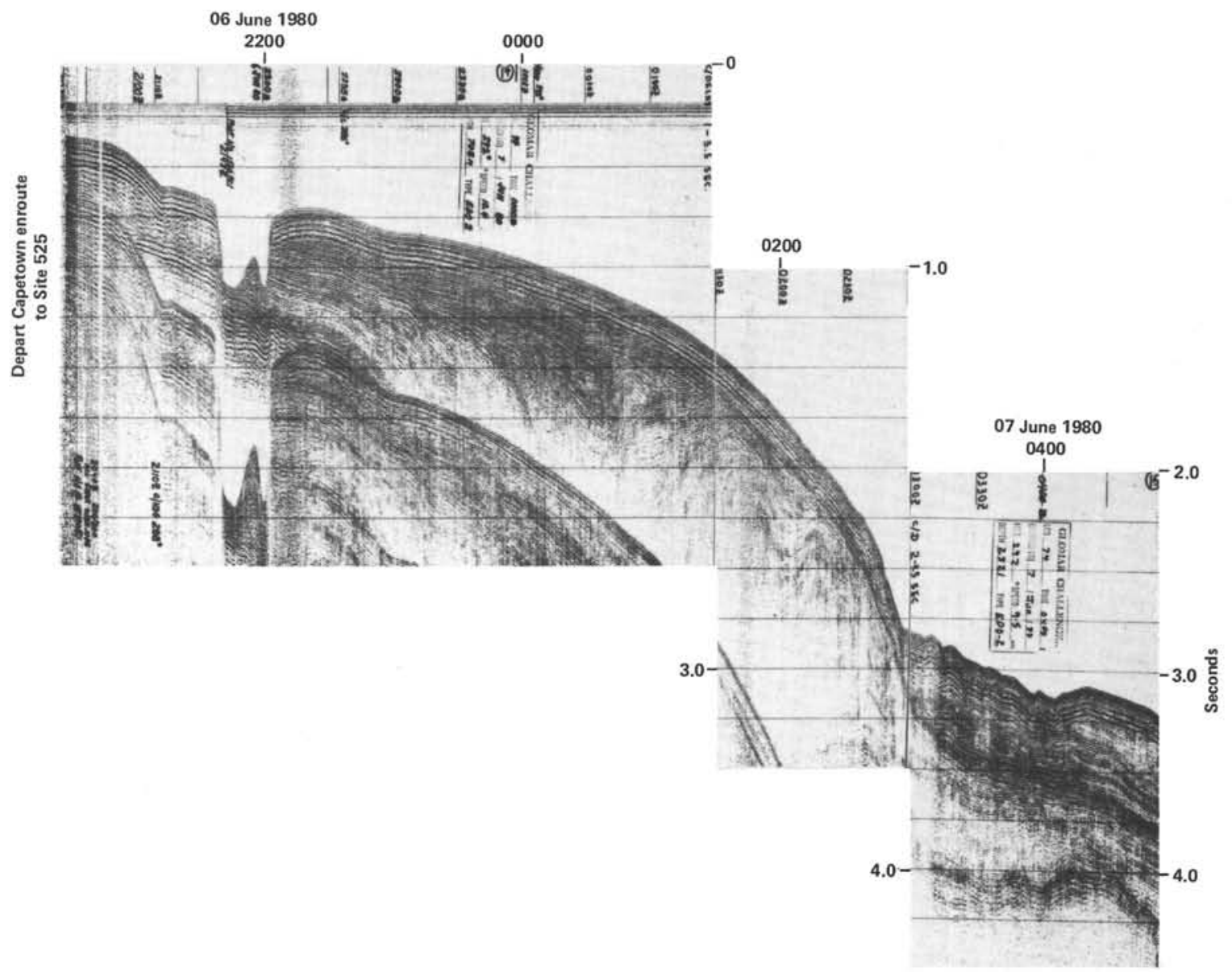

Figure 3. Seismic reflection profiles along tracks of Leg 74 of Glomar Challenger. 

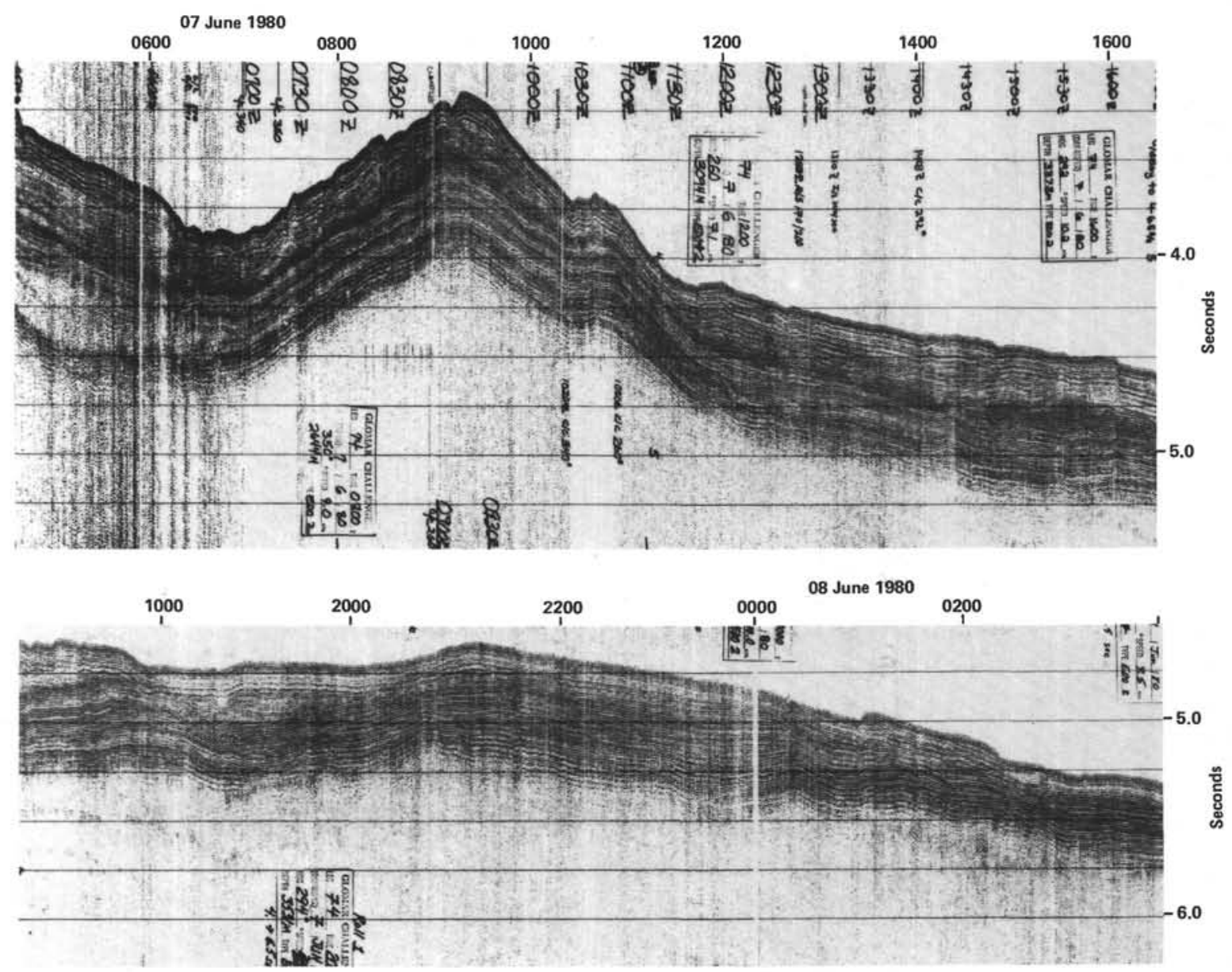

Figure 3. (Continued). 

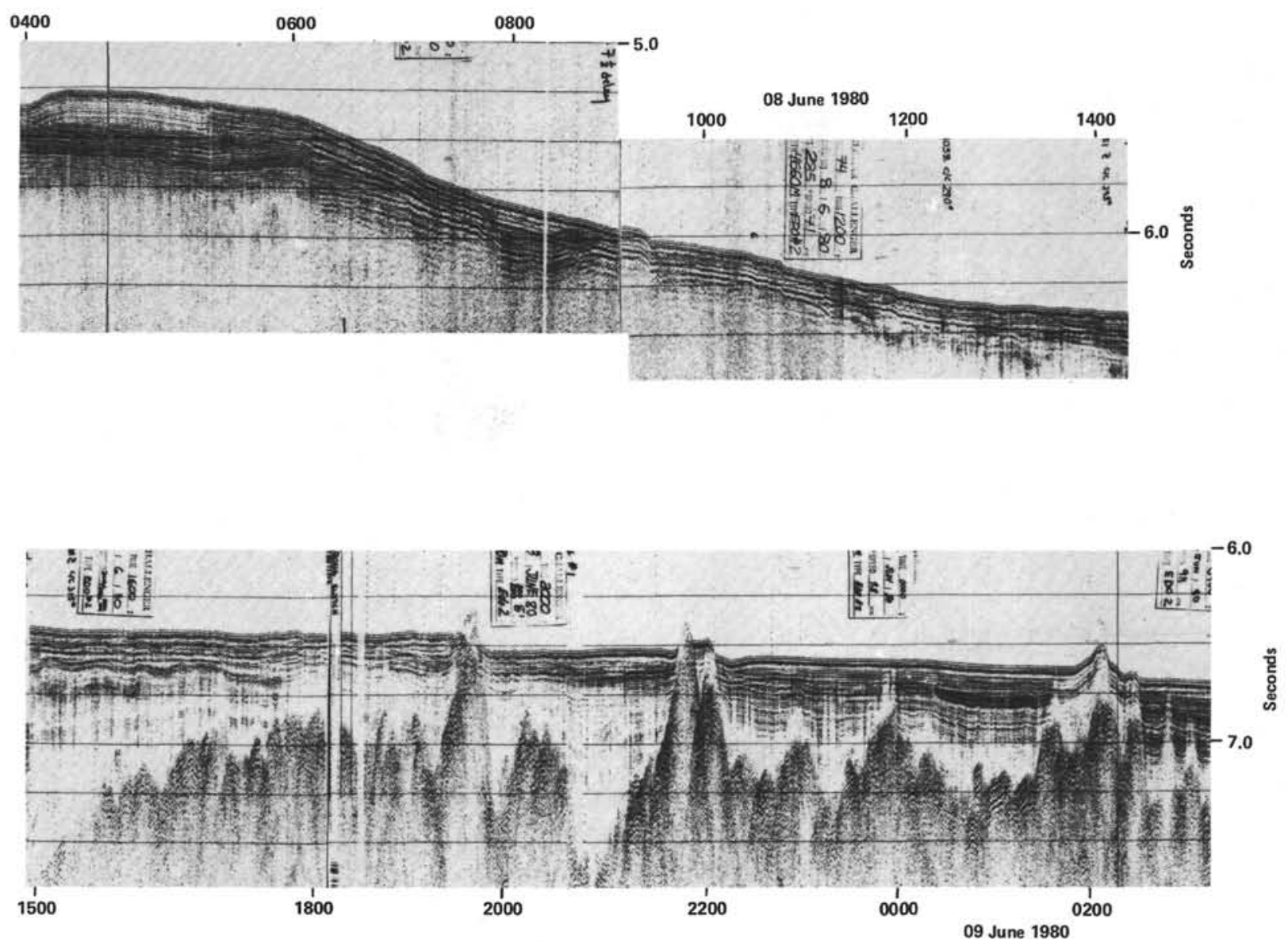

Figure 3. (Continued). 

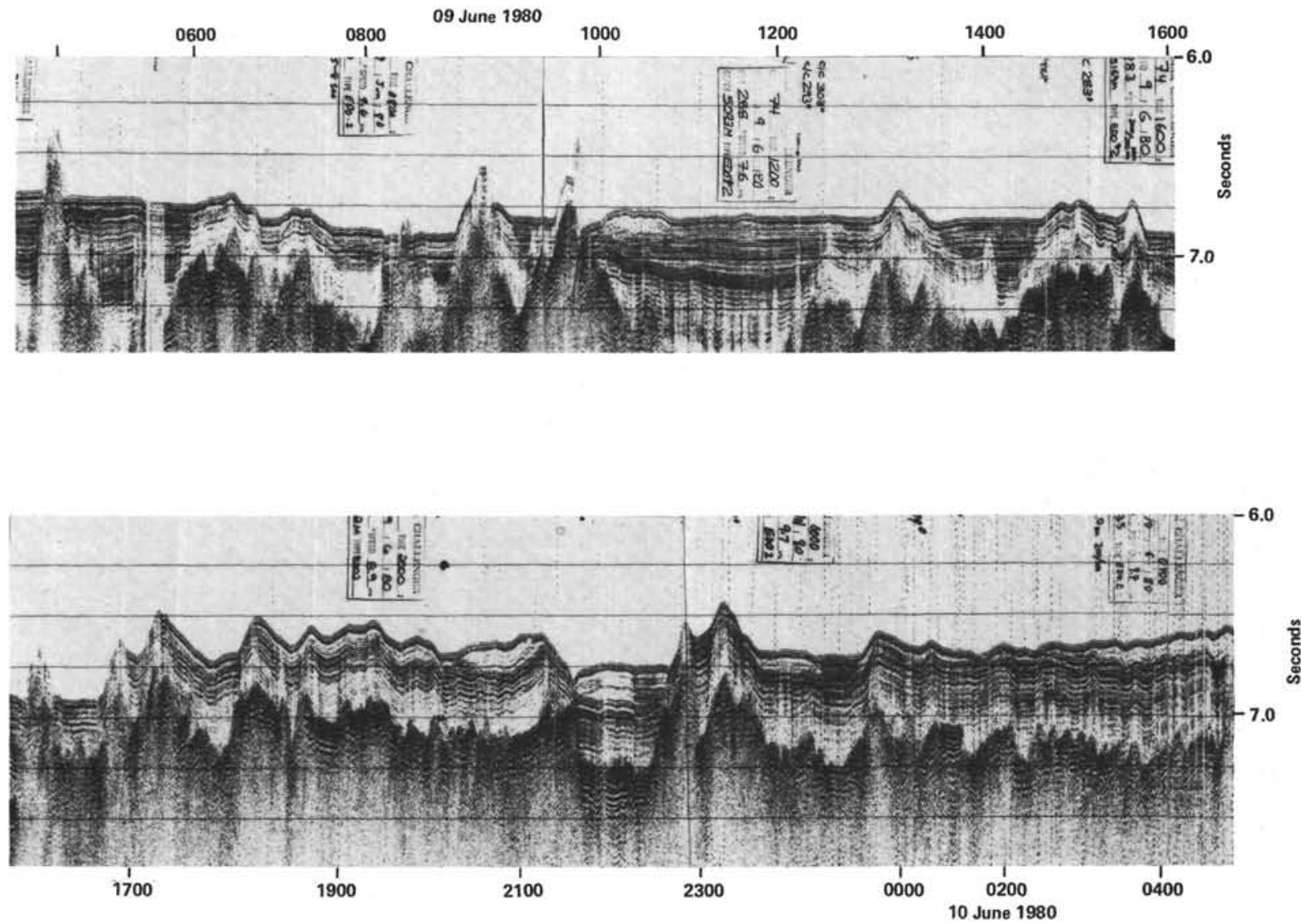

Figure 3. (Continued). 
P. D. RABINOWITZ, T. C. MOORE, JR., P. E. BORELLA, T. D. AITKEN
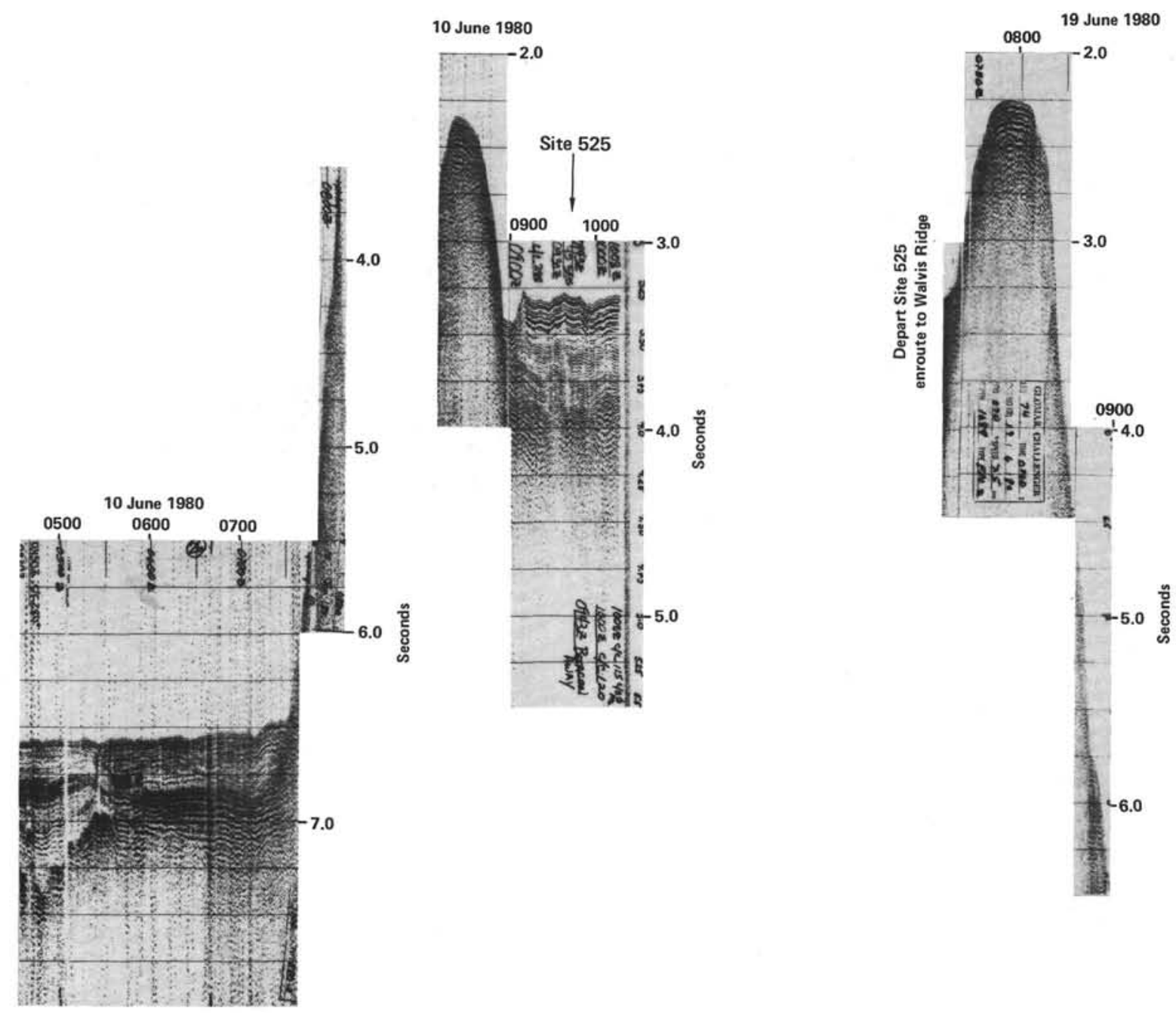

Figure 3. (Continued). 


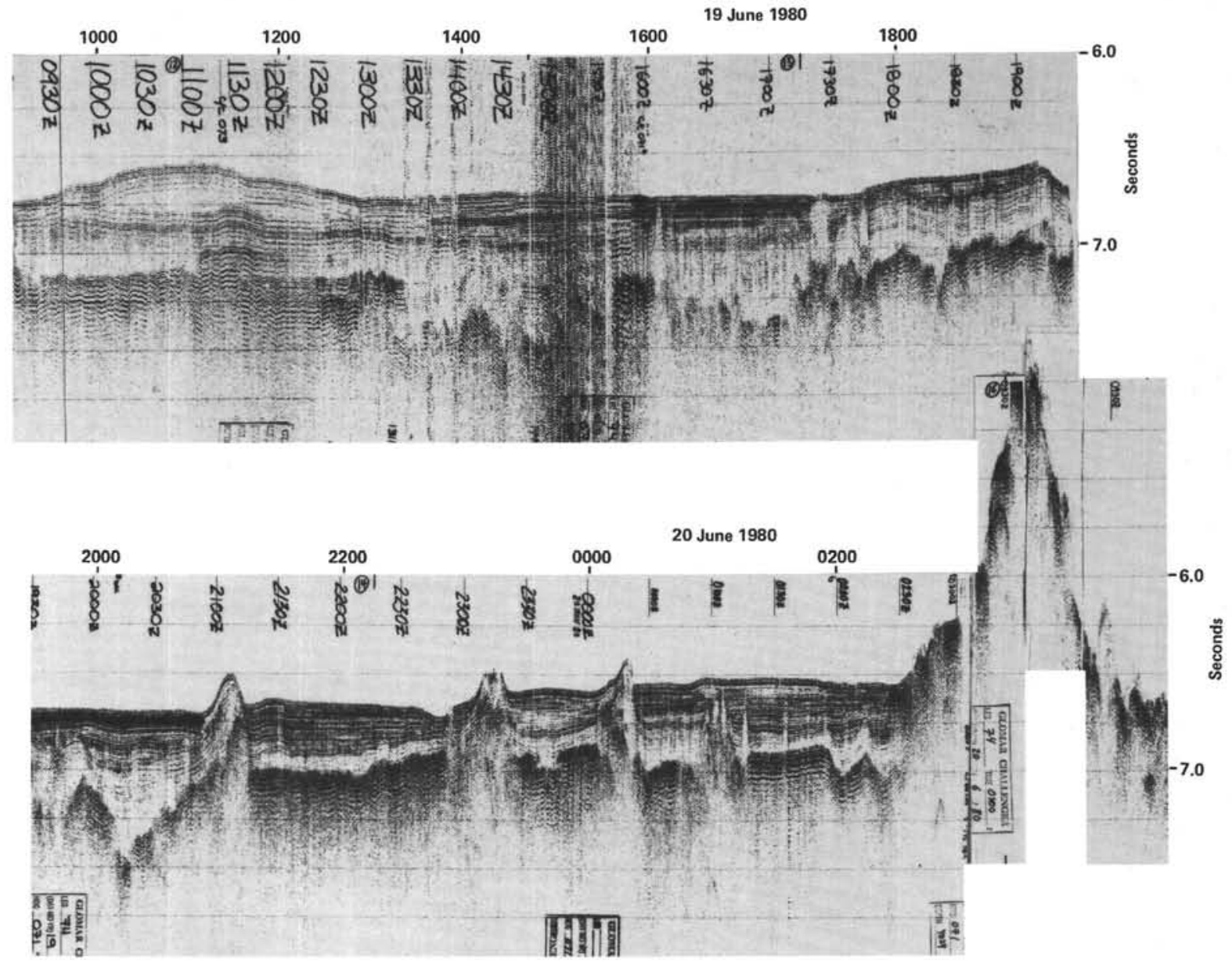

Figure 3. (Continued), 

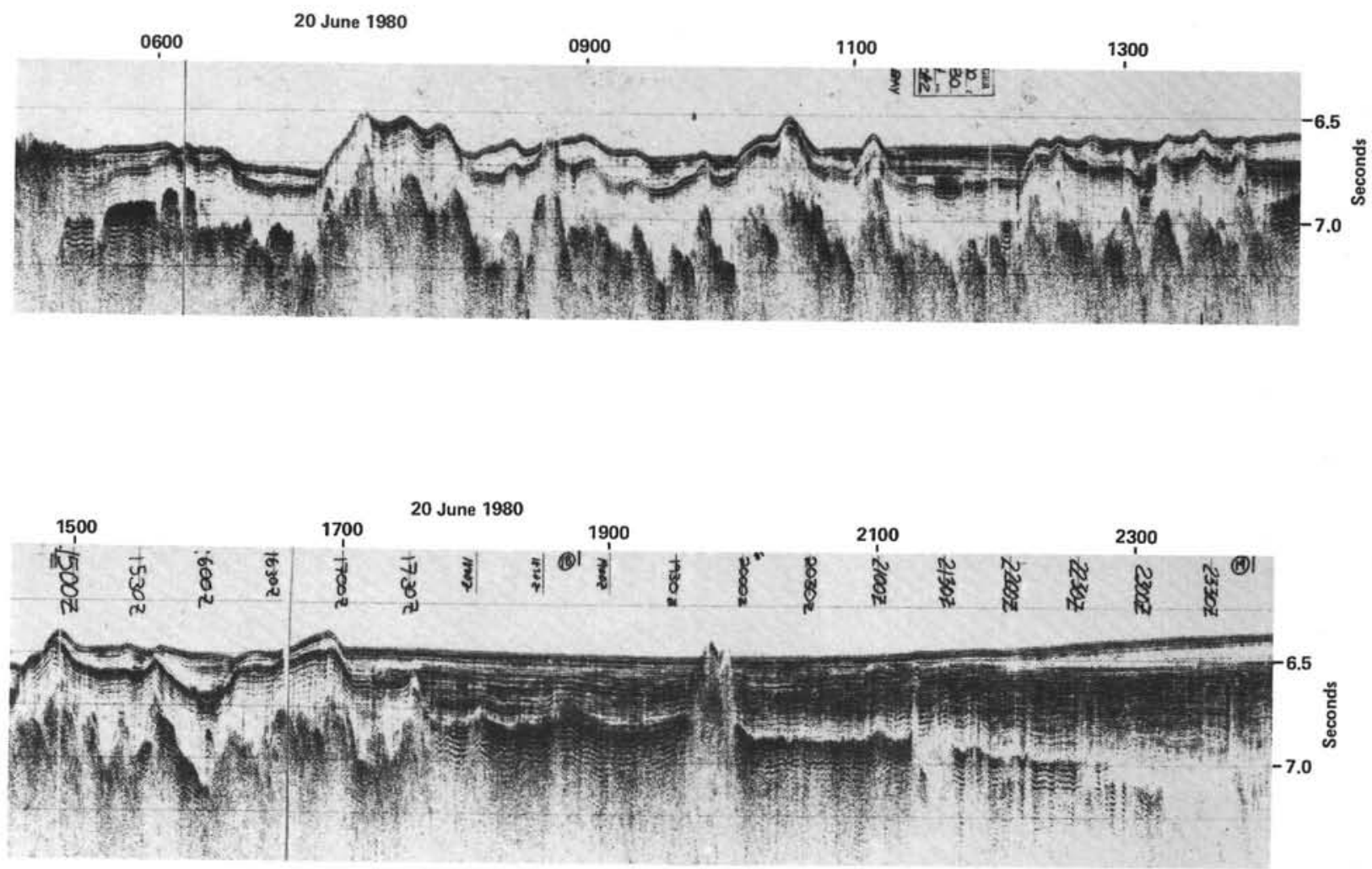

Figure 3. (Continued).

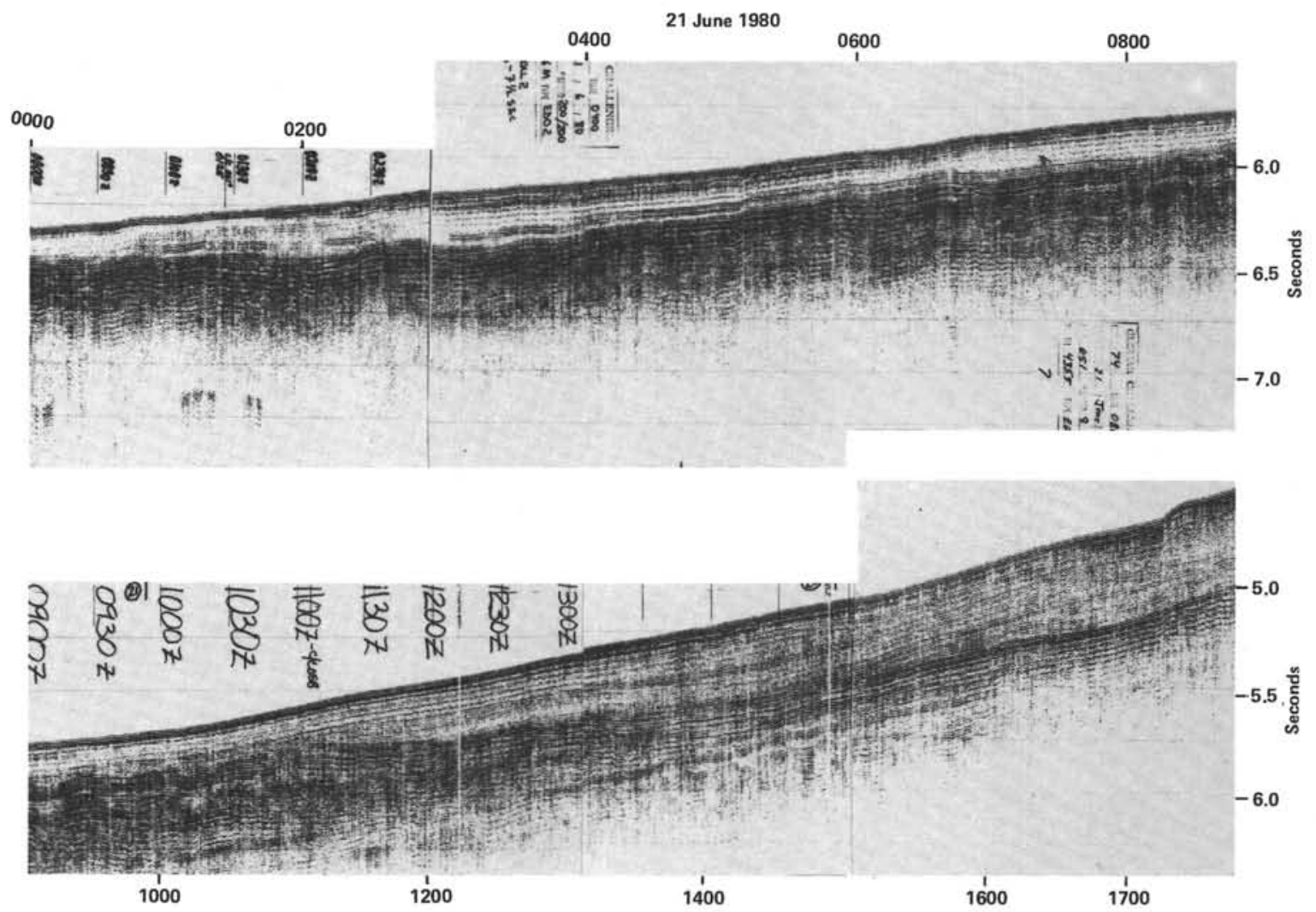

Figure 3. (Continued). 

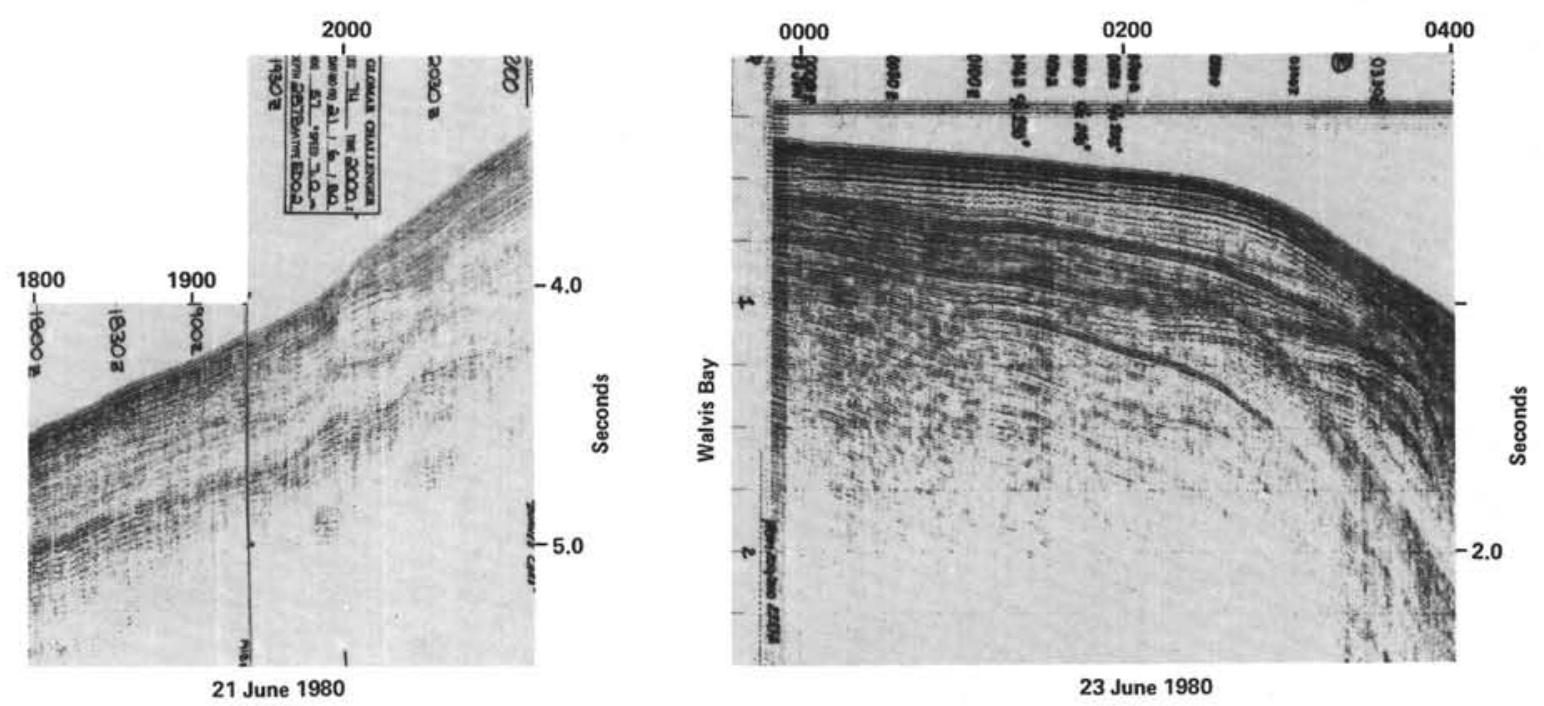

Figure 3. (Continued).

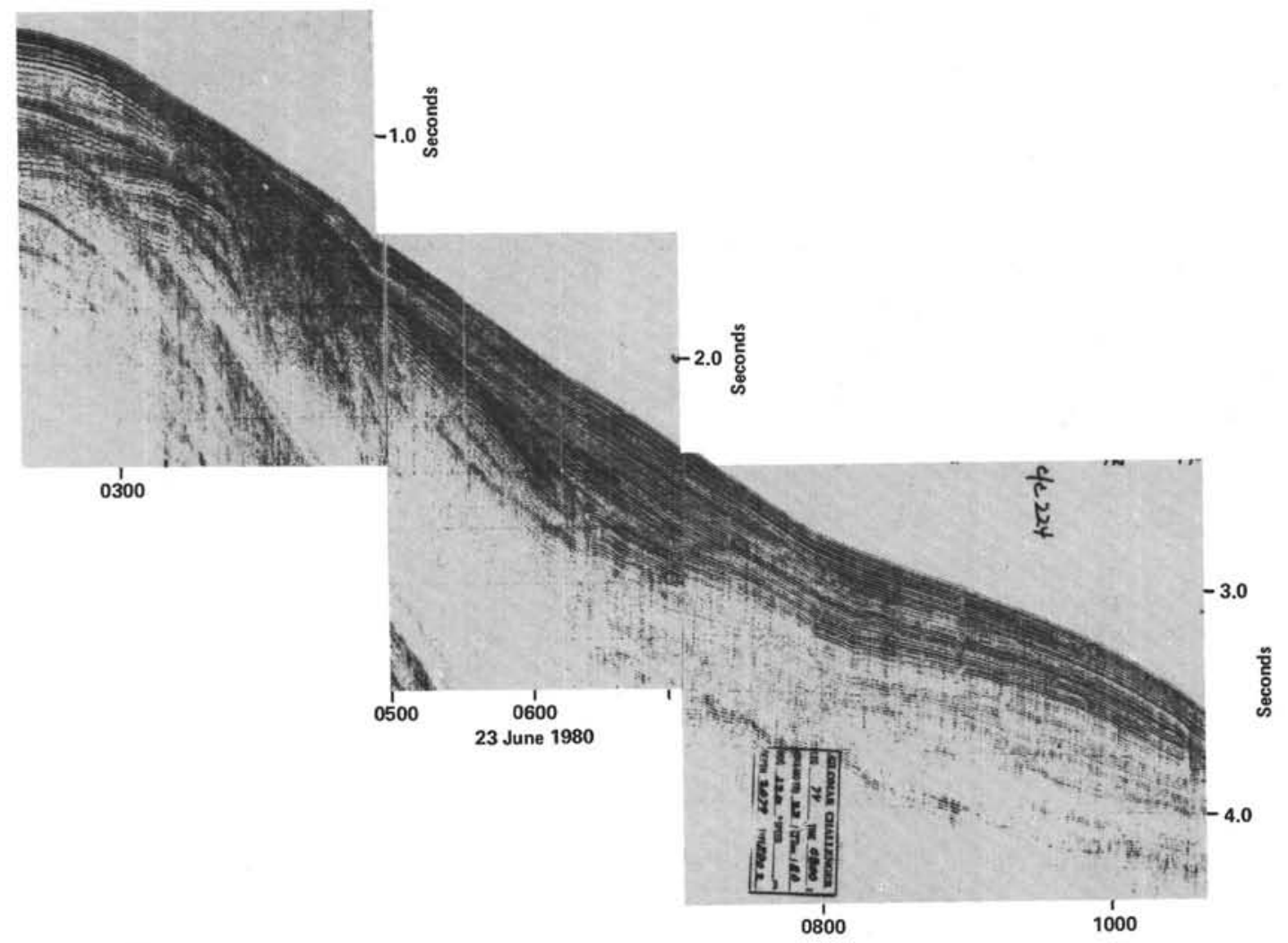

Figure 3. (Continued). 

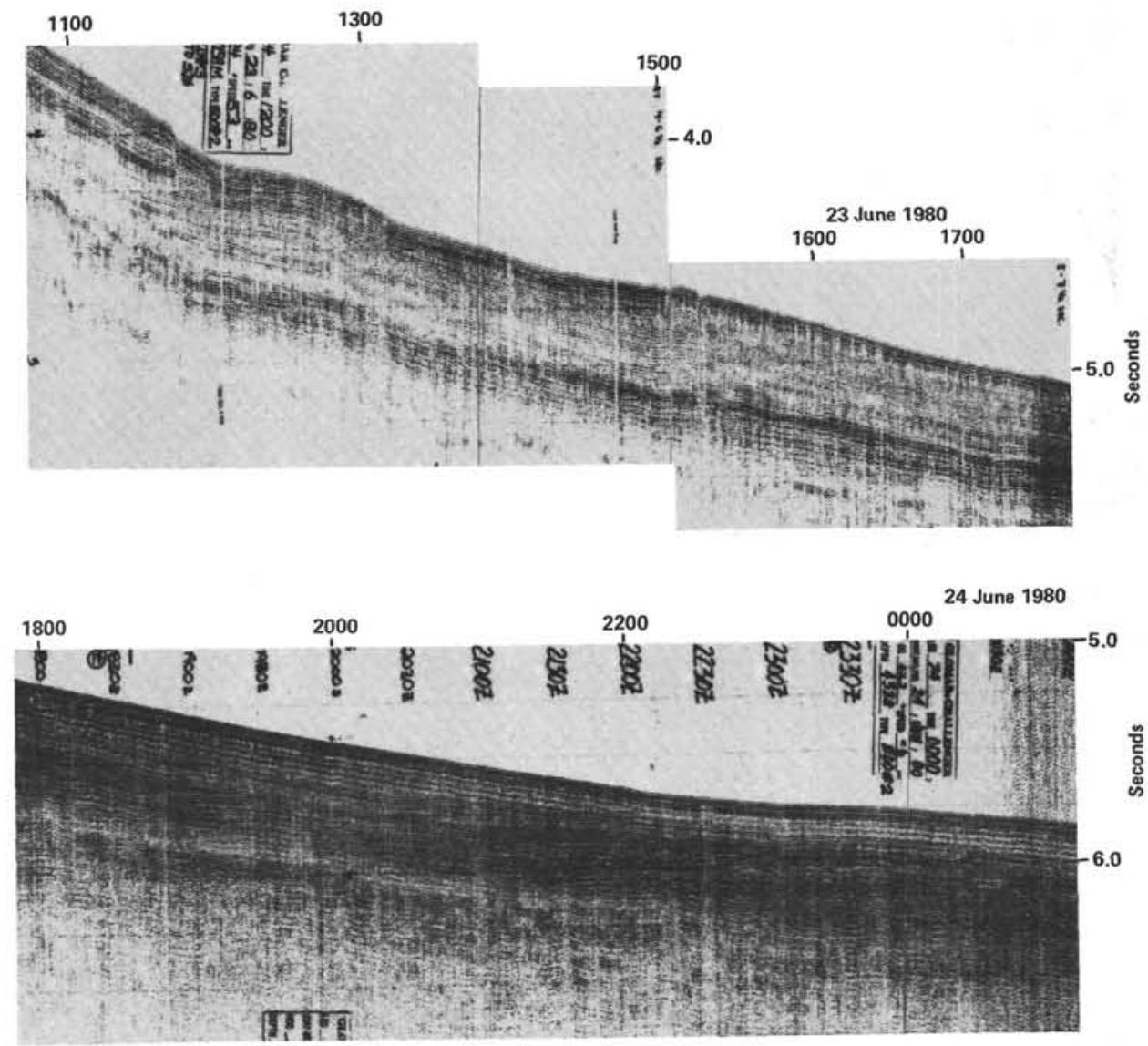

Figure 3. (Continued). 

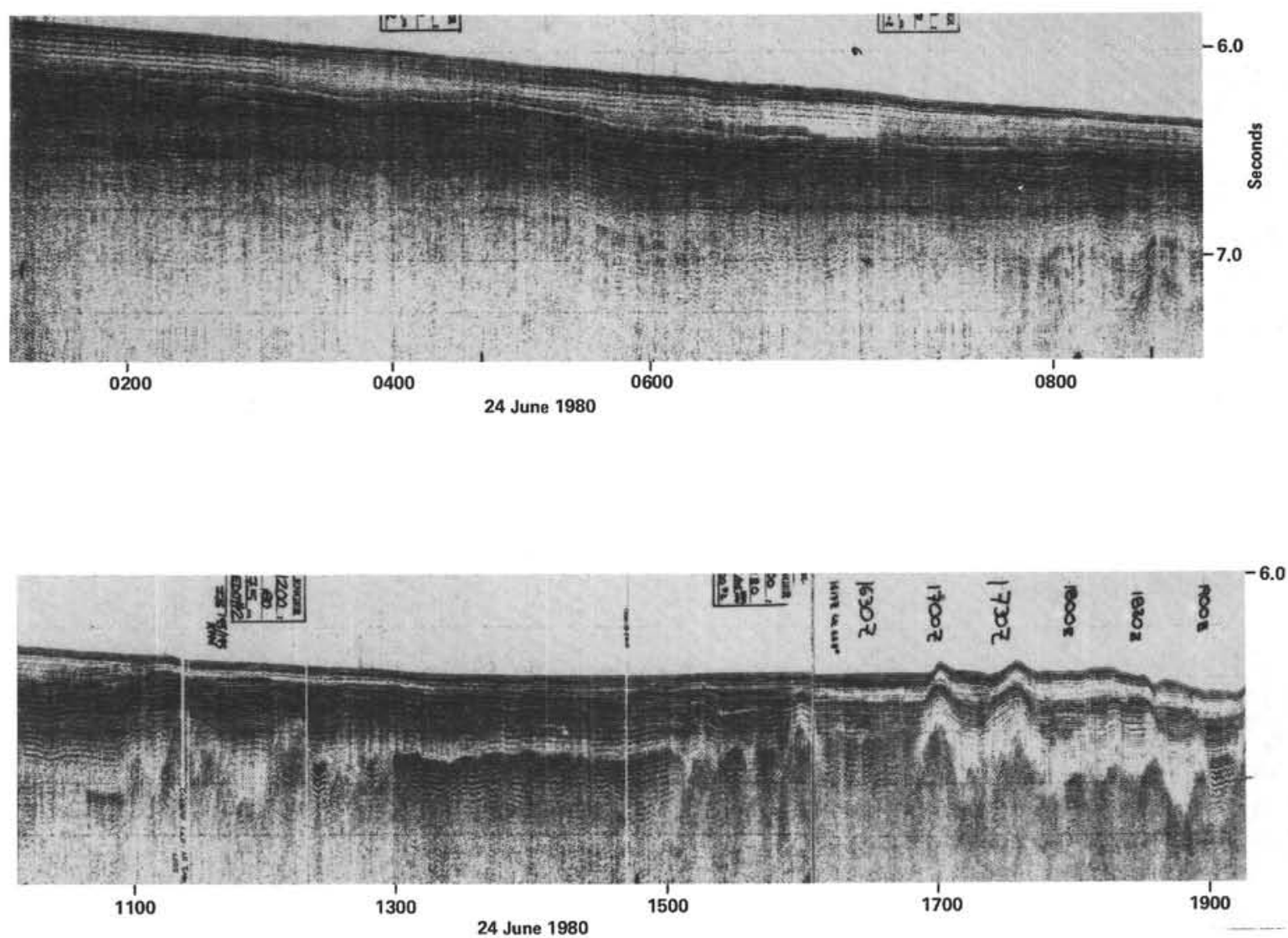

Figure 3. (Continued). 

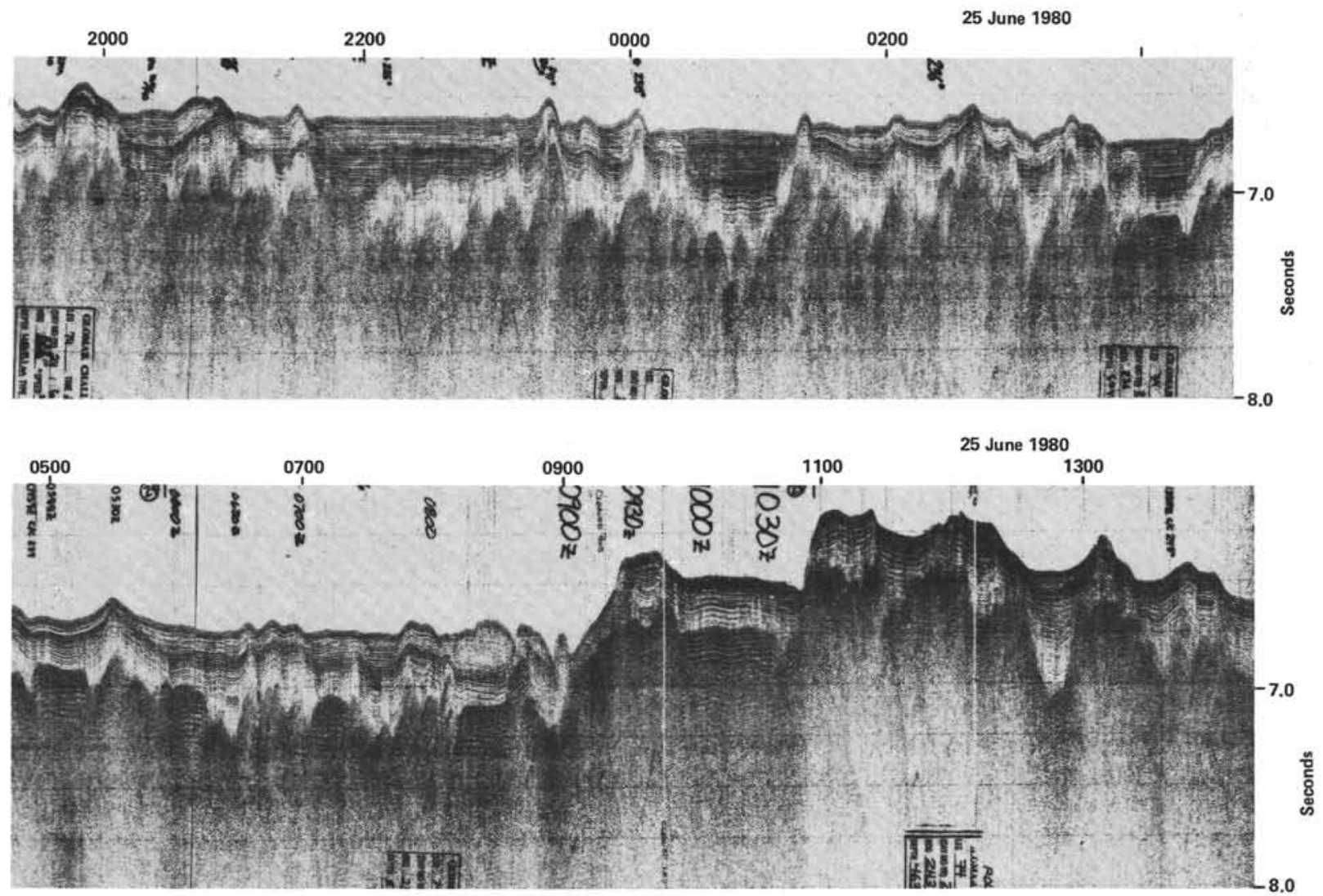

Figure 3. (Continued). 


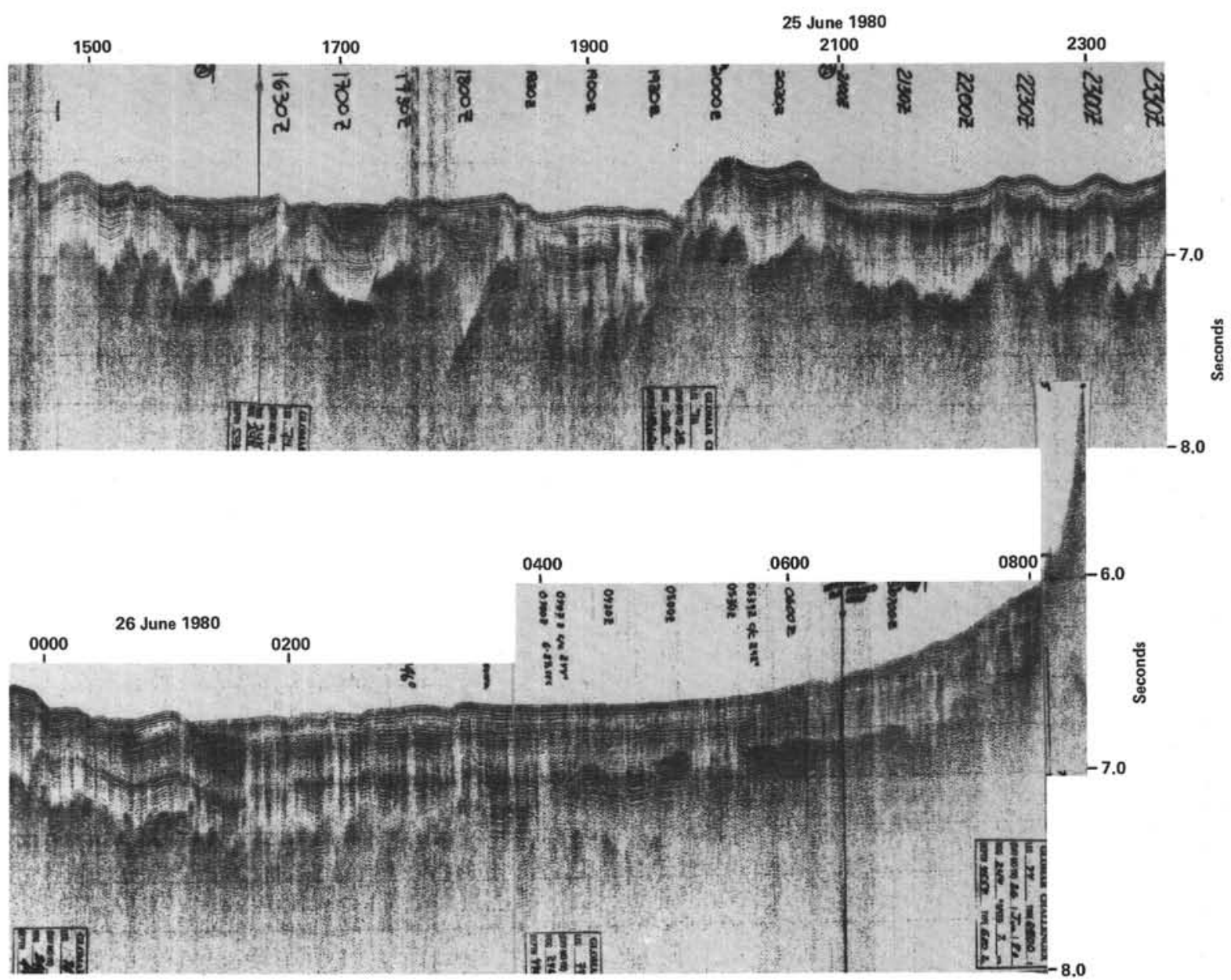

Figure 3. (Continued). 
P. D. RABINOWITZ, T. C. MOORE, JR., P. E. BORELLA, T. D. AITKEN
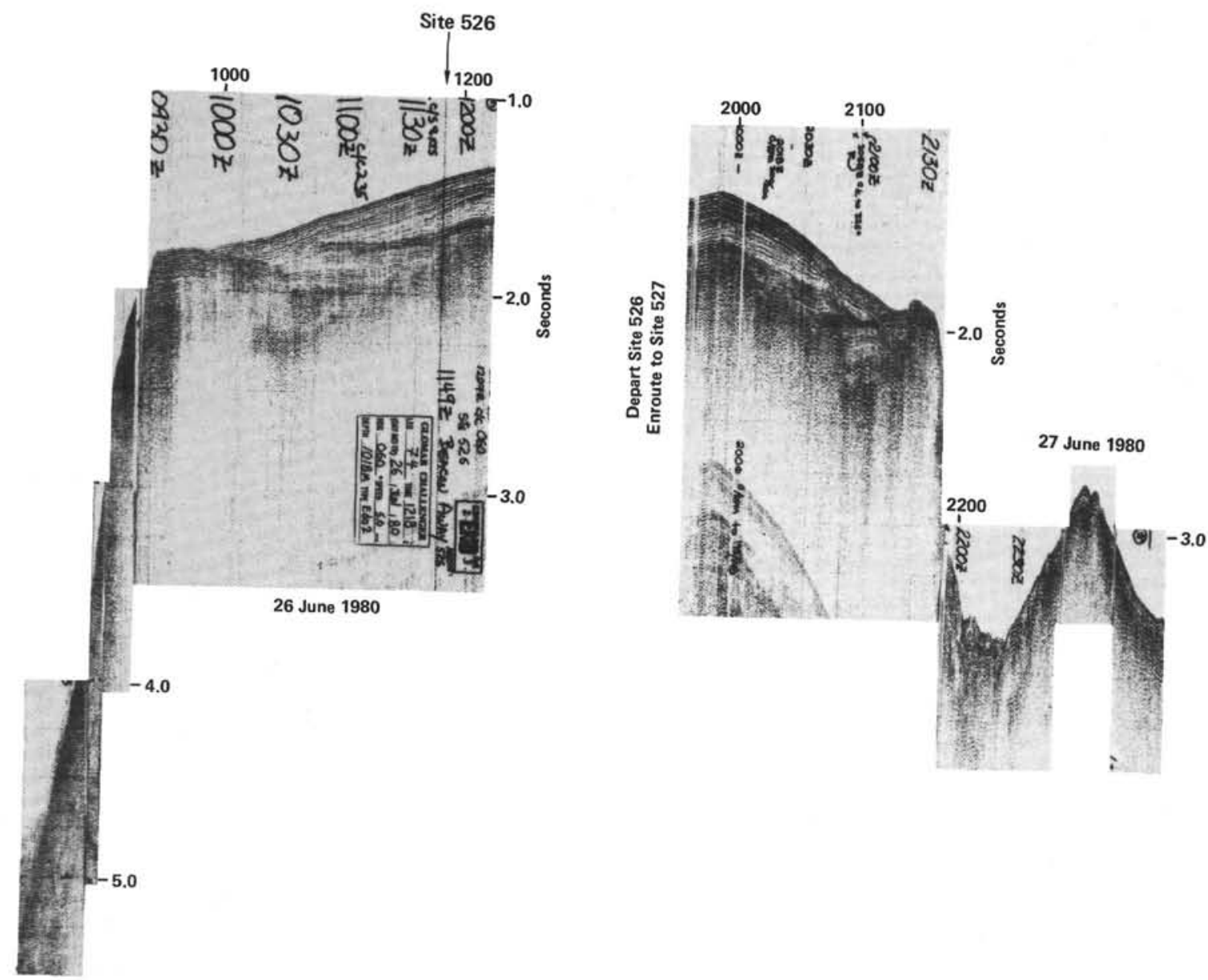

Figure 3. (Continued). 


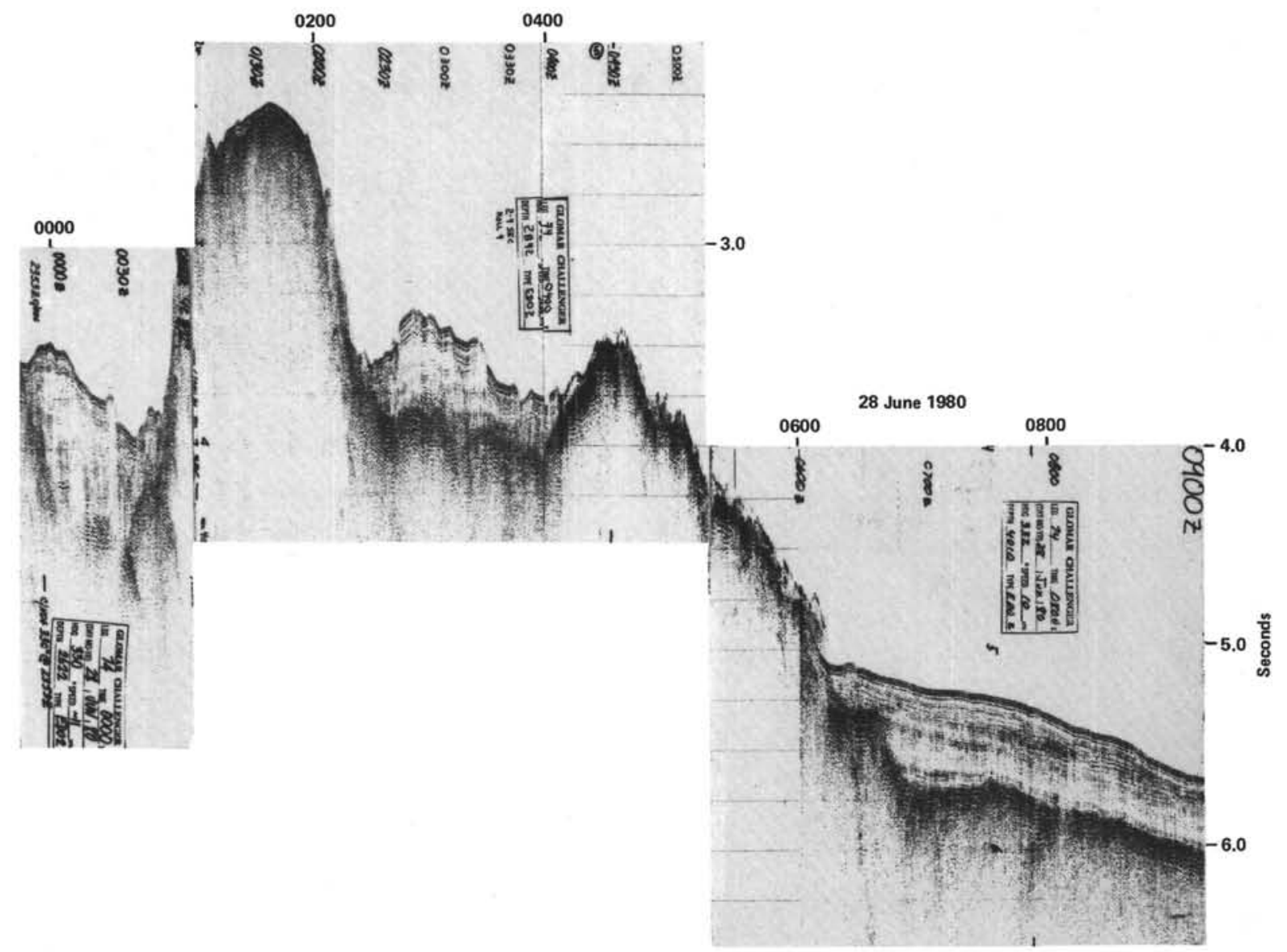

Figure 3. (Continued). 
P. D. RABINOWITZ, T. C. MOORE, JR., P. E. BORELLA, T. D. AITKEN
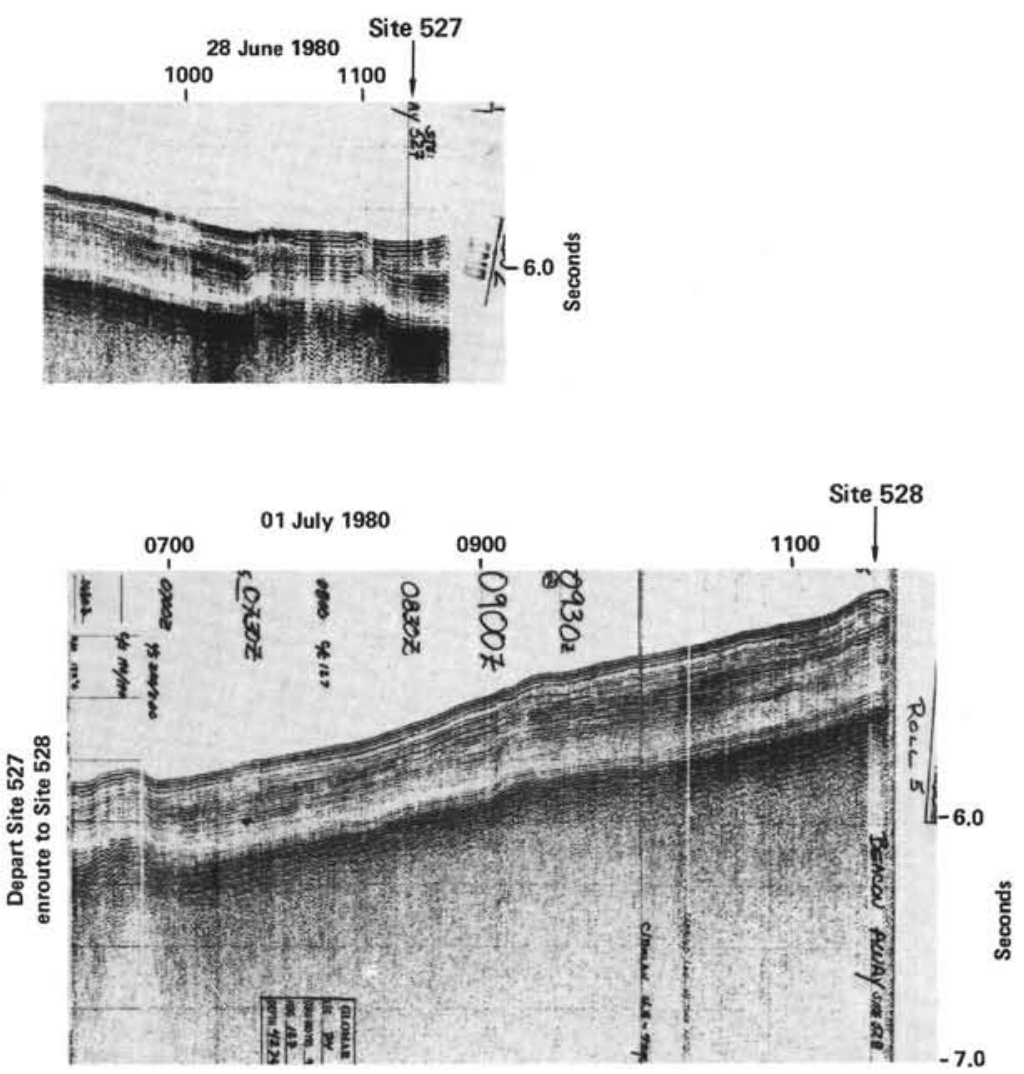

Figure 3. (Continued). 


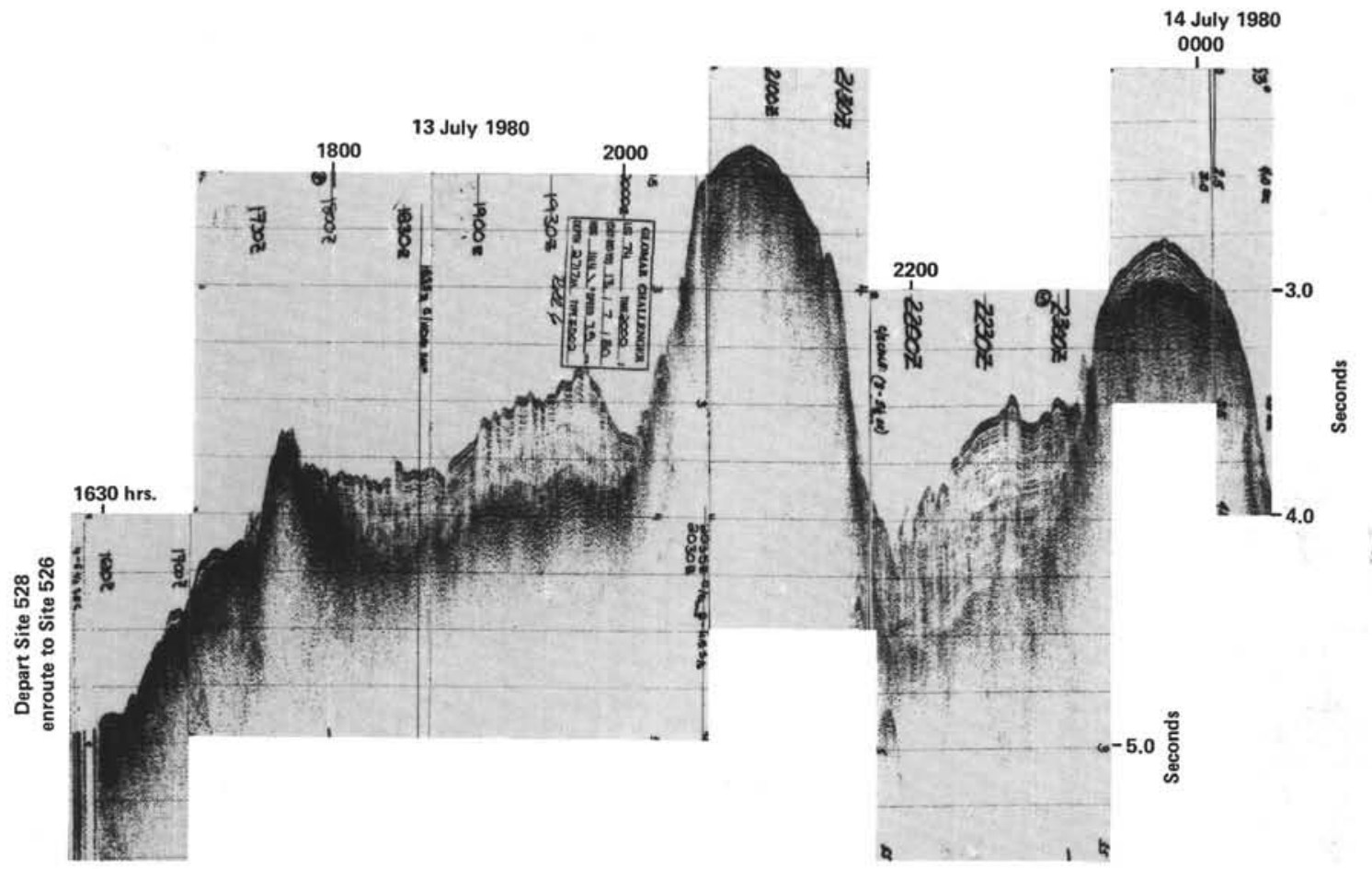

Figure 3. (Continued).
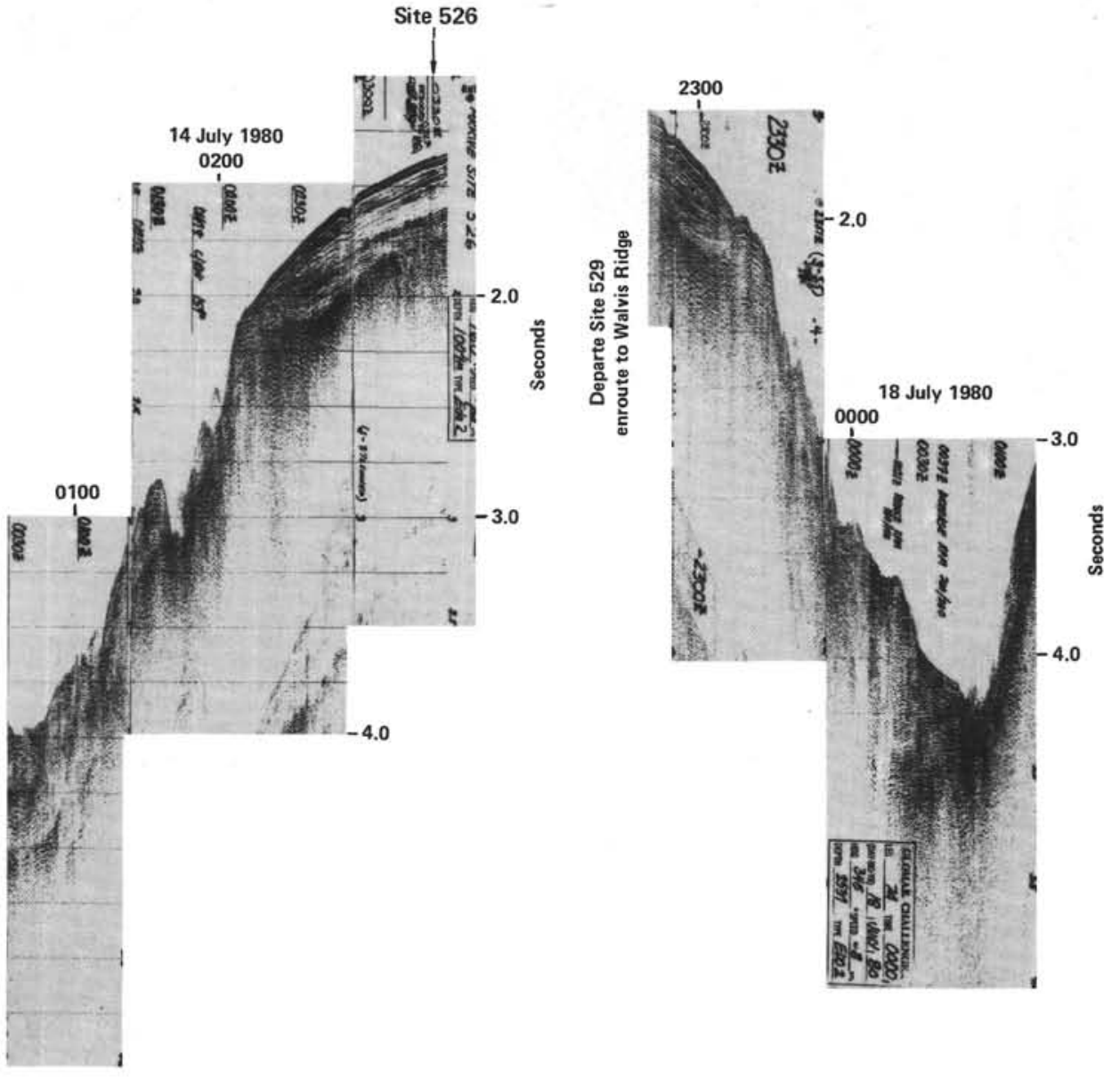

Figure 3. (Continued). 

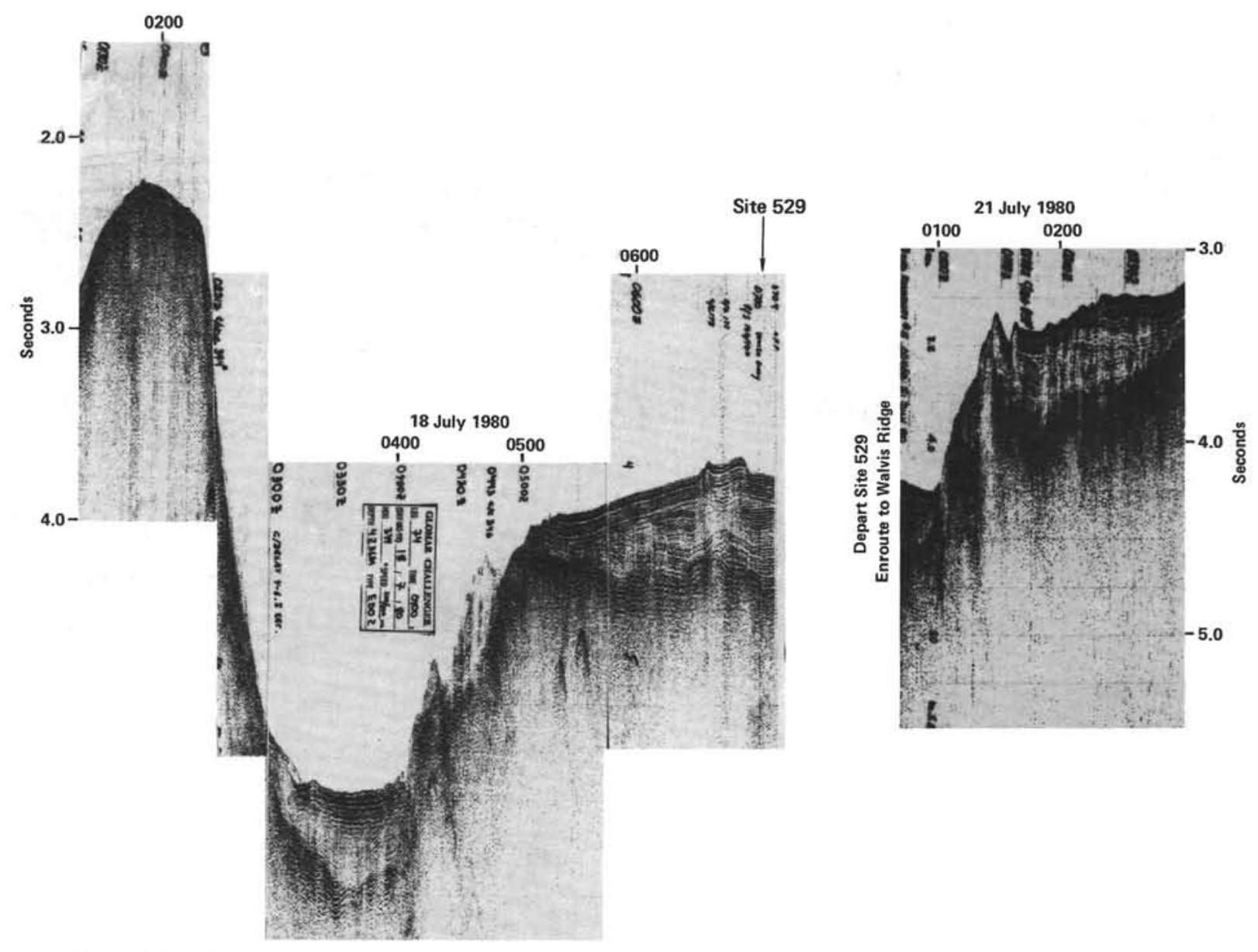

Figure 3. (Continued). 


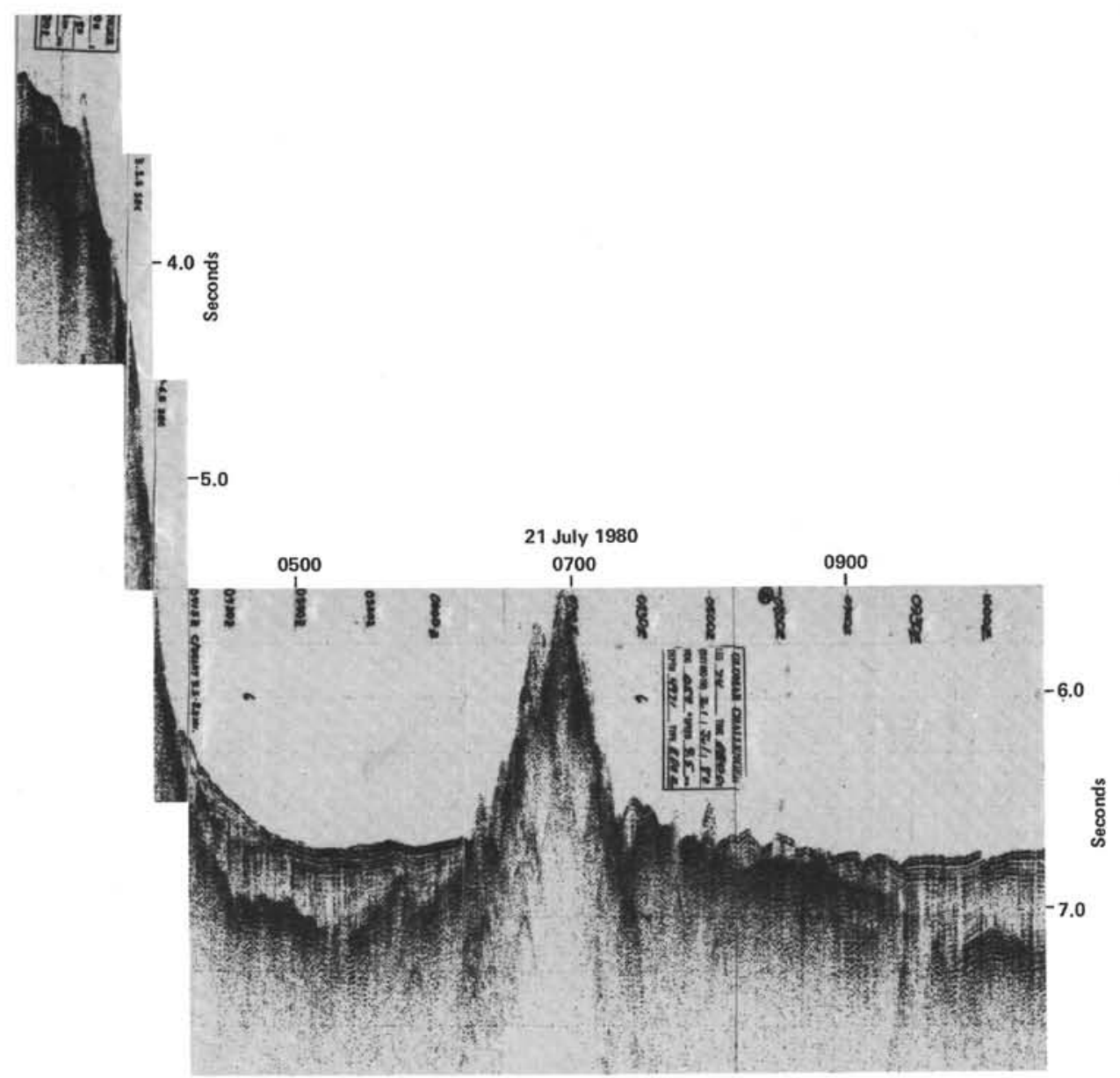

Figure 3. (Continued). 
P. D. RABINOWITZ, T. C. MOORE, JR., P. E. BORELLA, T. D. AITKEN
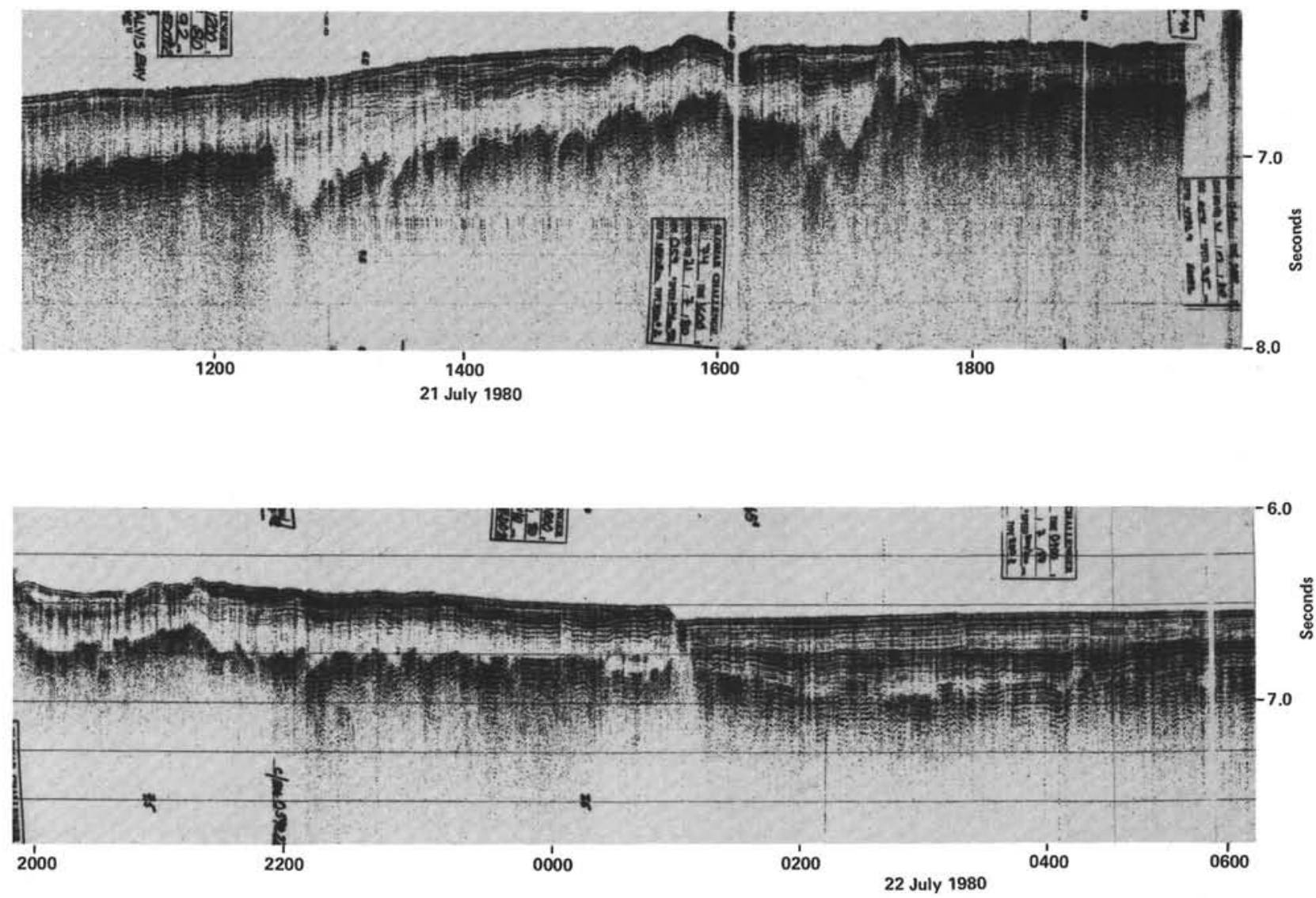

Figure 3. (Continued). 

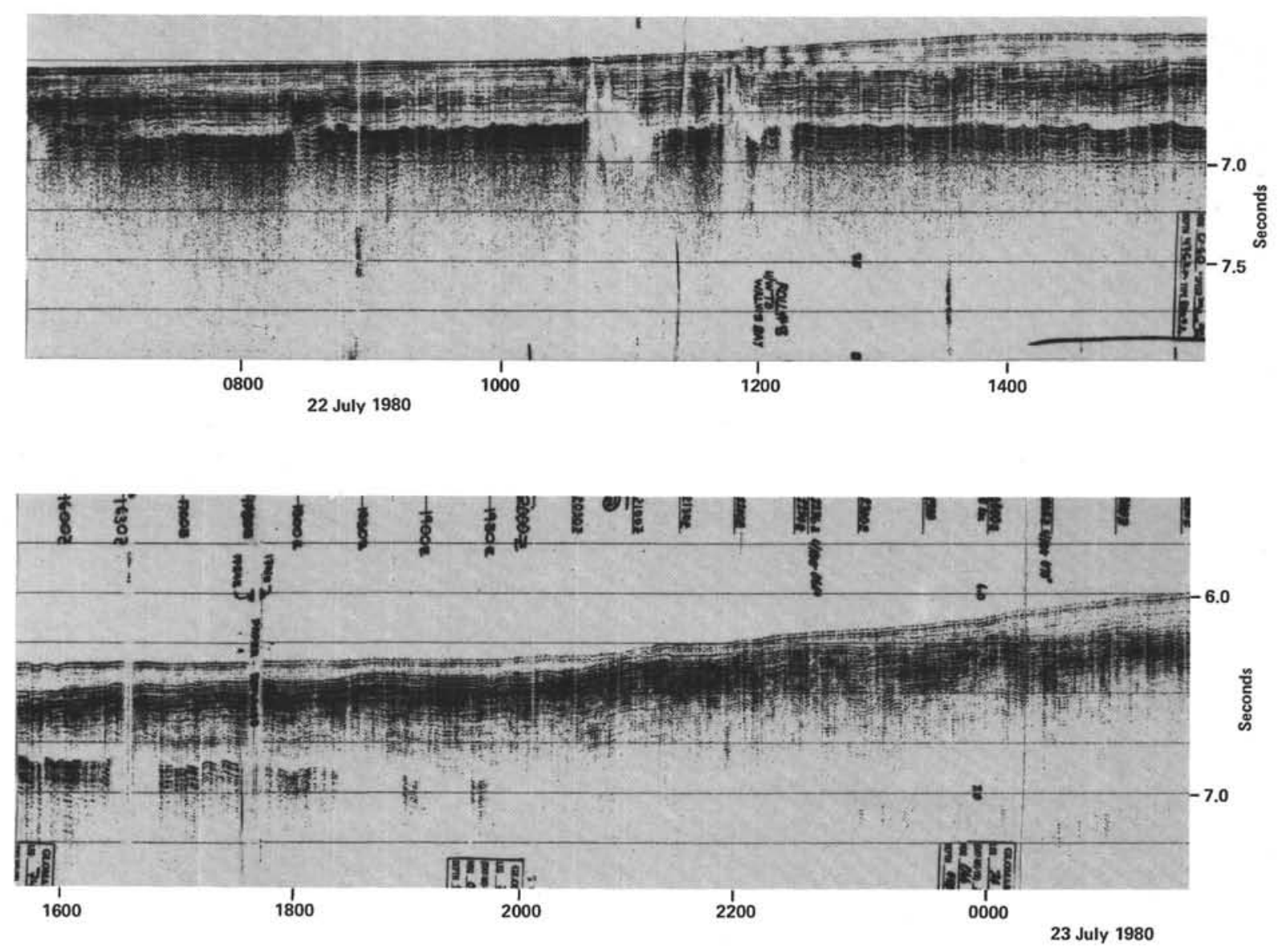

Figure 3. (Continued). 

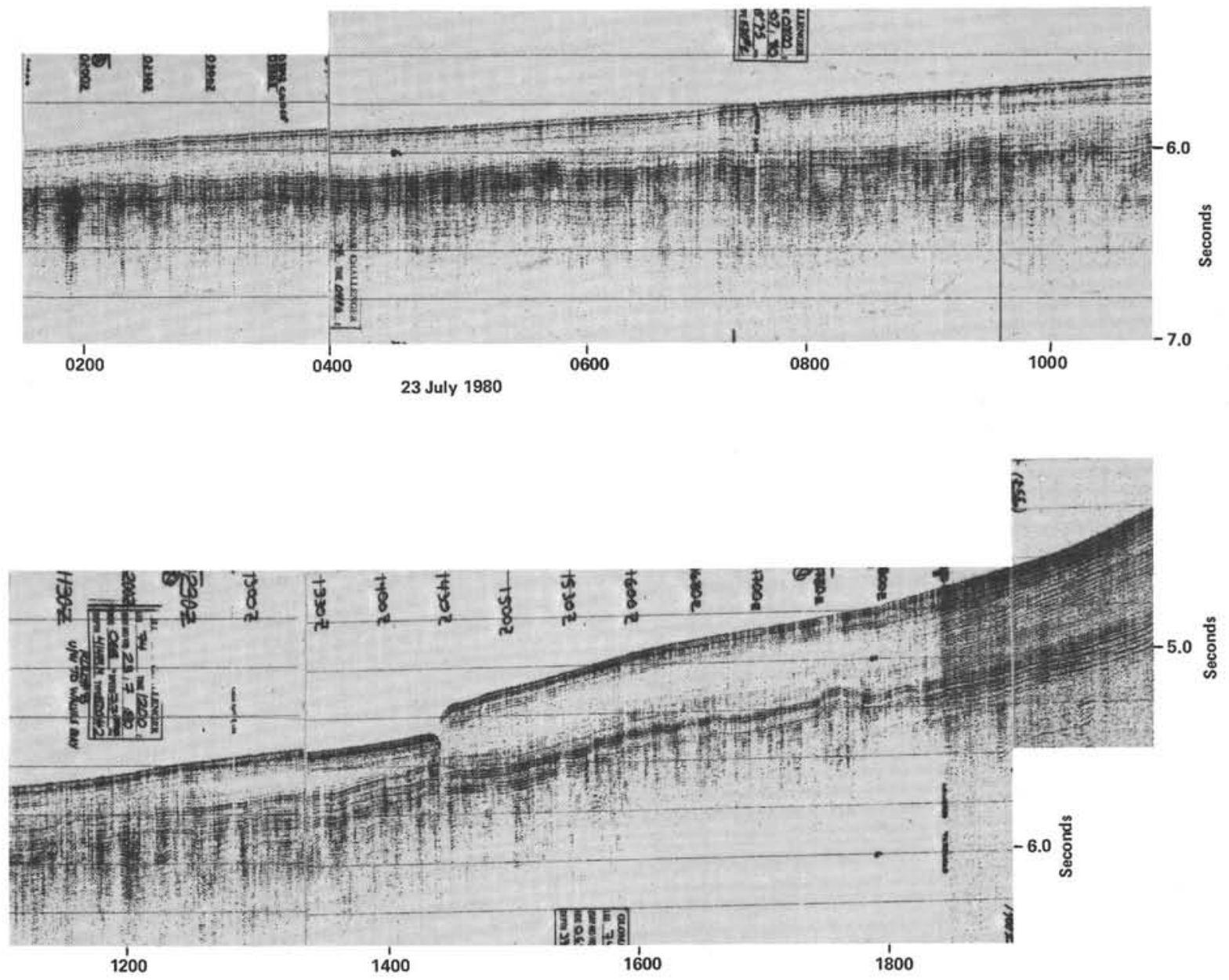

Figure 3. (Continued). 


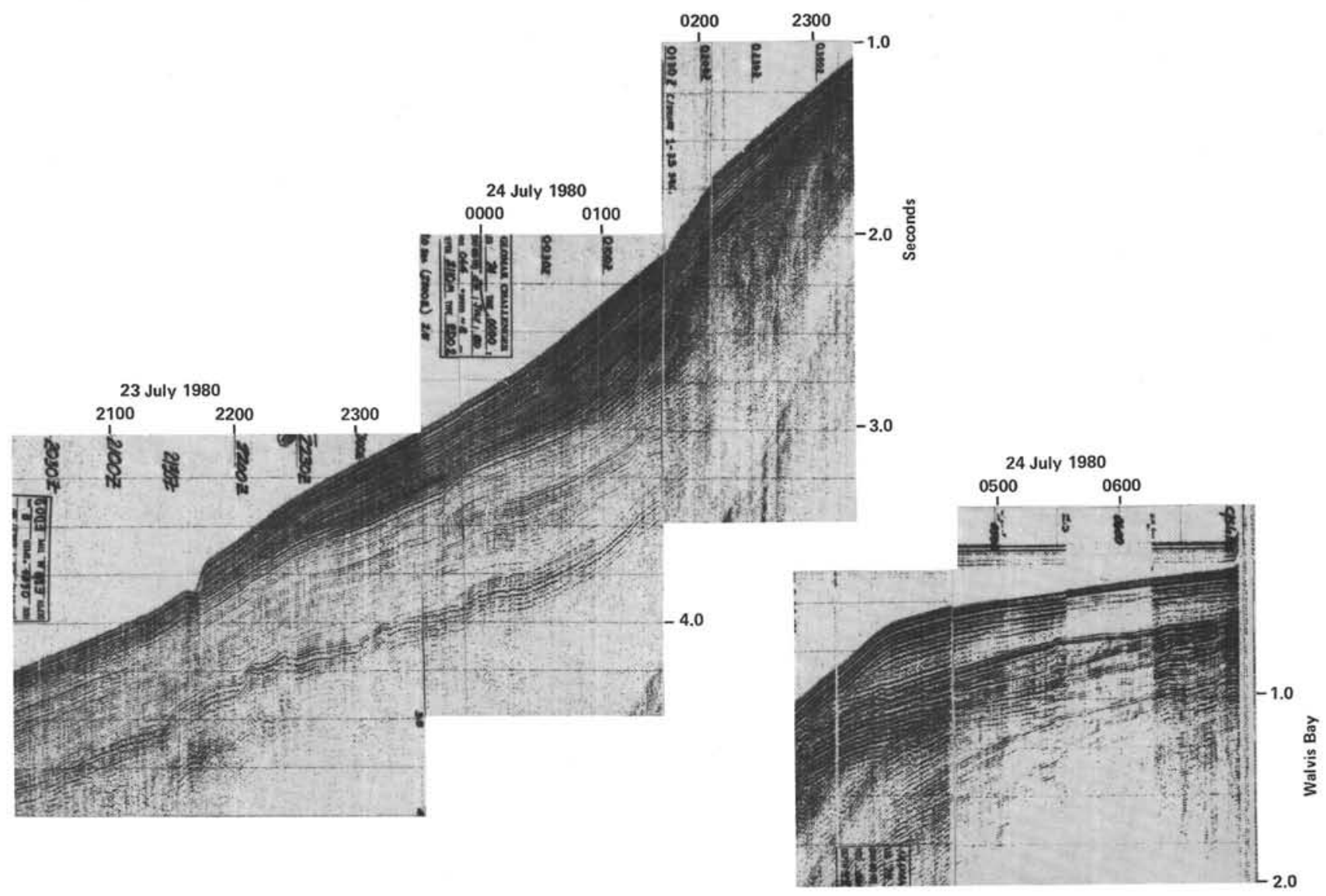

Figure 3. (Continued). 